\title{
Targeted Ablation of Oligodendrocytes Induces Axonal Pathology Independent of Overt Demyelination
}

\author{
Laura-Jane Oluich, ${ }^{1,2}$ Jo Anne S. Stratton, ${ }^{1,2}$ Yao Lulu Xing, ${ }^{1,2}$ Sze Woei Ng, ${ }^{1}$ Holly S. Cate, ${ }^{1,2}$ Pankaj Sah, ${ }^{3}$ \\ François Windels, ${ }^{3}$ Trevor J. Kilpatrick, ${ }^{1,2}$ and Tobias D. Merson ${ }^{1,2}$ \\ ${ }^{1}$ Florey Neuroscience Institutes, University of Melbourne, Parkville, Victoria 3010, Australia, ${ }^{2}$ Centre for Neuroscience Research, University of Melbourne, \\ Parkville, Victoria 3010, Australia, and ${ }^{3}$ Queensland Brain Institute, The University of Queensland, Brisbane, Queensland, 4072, Australia
}

The critical role of oligodendrocytes in producing and maintaining myelin that supports rapid axonal conduction in CNS neurons is well established. More recently, additional roles for oligodendrocytes have been posited, including provision of trophic factors and metabolic support for neurons. To investigate the functional consequences of oligodendrocyte loss, we have generated a transgenic mouse model of conditional oligodendrocyte ablation. In this model, oligodendrocytes are rendered selectively sensitive to exogenously administered diphtheria toxin (DT) by targeted expression of the diphtheria toxin receptor in oligodendrocytes. Administration of DT resulted in severe clinical dysfunction with an ascending spastic paralysis ultimately resulting in fatal respiratory impairment within $22 \mathrm{~d}$ of DT challenge. Pathologically, at this time point, mice exhibited a loss of $\sim 26 \%$ of oligodendrocyte cell bodies throughout the CNS. Oligodendrocyte cell-body loss was associated with moderate microglial activation, but no widespread myelin degradation. These changes were accompanied with acute axonal injury as characterized by structural and biochemical alterations at nodes of Ranvier and reduced somatosensory-evoked potentials. In summary, we have shown that a death signal initiated within oligodendrocytes results in subcellular changes and loss of key symbiotic interactions between the oligodendrocyte and the axons it ensheaths. This produces profound functional consequences that occur before the removal of the myelin membrane, i.e., in the absence of demyelination. These findings have clear implications for the understanding of the pathogenesis of diseases of the CNS such as multiple sclerosis in which the oligodendrocyte is potentially targeted.

\section{Introduction}

The oligodendrocyte is a specialized glial cell within the CNS that is responsible for producing the myelin that ensheaths axons and forms the nodes of Ranvier that permit saltatory nerve conduction. Dysfunction and death of oligodendrocytes has been described in various demyelinating pathologies including the leukodystrophies and multiple sclerosis (MS) (Lucchinetti et al., 1996; Dowling et al., 1999; Feigenbaum et al., 2000). Autoimmune-induced inflammatory demyelination has traditionally been the assumed pathogenic mechanism in MS (Weiner, 2004; Frohman et al., 2006). However, recent detailed examinations of pathological specimens have challenged this view. On the one hand, significant axonal degeneration occurs early in disease (Trapp et al., 1998; 1999; Kuhlmann et al., 2002),

\footnotetext{
Received March 2, 2012; revised March 22, 2012; accepted March 28, 2012

Author contributions: H.S.C., P.S., T.J.K., and T.D.M. designed research; L.-J.O., J.A.S.S., Y.L.X., S.W.N., F.W., and T.D.M. performed research; L.-J.O. and T.D.M. analyzed data; T.J.K. and T.D.M. wrote the paper.

This work was supported by the Australian Postgraduate Award and Rotary Health Scholarship (L.J.0), the National Health and Medical Research Council of Australia (NHMRC), Multiple Sclerosis Research Australia (MSRA) Betty Cuthbert Fellowship (T.D.M.), NHMRC and MSRA Project Grants (T.D.M.), and an NHMRC Program Grant (T.J.K. and P.S.). Anna Friedhuber and Dennis Kemper provided technical assistance with electron microscopy. Charityworks for MS and the Victorian Operational Infrastructure Support Program provided additional support.

The authors declare no competing financial interests.

Correspondence should be addressed to either Tobias D. Merson or Trevor J. Kilpatrick, Multiple Sclerosis Division, Kenneth Myer Building, University of Melbourne, Parkville, Victoria 3010, Australia. E-mail: tmerson@unimelb.edu.au or tkilpat@unimelb.edu.au.

DOI:10.1523/JNEUROSCI.1053-12.2012

Copyright $\odot 2012$ the authors $\quad 0270-6474 / 12 / 328317-14 \$ 15.00 / 0$
}

even in the absence of demyelination (Nikić et al., 2011), raising the possibility of axonal attack by activated microglia and/or macrophages. On the other hand, oligodendrocyte death in acute MS can occur without significant immune cell infiltration and may precede the development of inflammatory demyelinated plaques (Barnett and Prineas, 2004; Marik et al., 2007; Henderson et al., 2009). Moreover, periplaque tissue in evolving MS lesions exhibits oxidative damage that is often localized within apoptotic oligodendrocytes (Haider et al., 2011). Thus, oligodendrocytopathy could represent the primary event in newly forming MS lesions.

Experimental models of primary oligodendrocytopathy could assist in unraveling the complex pathological sequelae in MS. To this end, specific oligodendrocyte-targeting models have recently been developed using diphtheria toxin (DT) as the driver of oligodendrocyte death (Buch et al., 2005; Traka et al., 2010; Pohl et al., 2011). These binary genetic approaches involve oligodendrocyte-restricted Cre or CreER-regulated expression of the simian DT receptor (DTR) or the A subunit of DT (DT-A). In the former scenario, expression of DTR in oligodendrocytes renders naturally DT-resistant mouse cells susceptible to intraperitoneally injected DT. Once internalized, DT catalyzes ADPribosylation of eukaryotic elongation factor 2, which results in inhibition of protein translation and ultimately, apoptosis (Collier, 2001). Induction of oligodendrocyte apoptosis in these mice causes demyelination, myelin splitting, and/or vacuolization resulting in motor pathologies of variable severity (Buch et al., 2005; Traka et al., 2010; Pohl et al., 2011). 
We have generated a novel transgenic mouse denoted MBPDTR (Line 100A) for targeted ablation of oligodendrocytes that does not rely upon Cre/loxP transgenics. In these mice, expression of human DTR is regulated by the proximal $1.96 \mathrm{~kb}$ promoter of mouse myelin basic protein $(M B P)$ gene which directs specific expression to mature oligodendrocytes (Gow et al., 1992; Shults et al., 2005; Emery et al., 2006). Administration of DT to MBP-DTR mice leads to loss of oligodendrocyte cell bodies and the development of severe clinical pathology characterized by spastic paralysis of the hindlimbs, tremor, ataxia, and kyphosis that correlates with impairment of electrophysiological properties of neurones and ultrastructural alterations at the nodes of Ranvier. Importantly, these changes occurred in the absence of inflammatory demyelination and demonstrate that axonal pathology occurring in the absence of demyelination can still be secondary to primary pathology of the oligodendrocyte.

\section{Materials and Methods}

Generation of MBP-DTR transgenic mice. The MBP-DTR construct was generated by excising a $L a c Z$ transgene from the pMG2 vector (kindly provided by Associate Professor Alexander Gow, Wayne State University, Detroit, Michigan; Gow et al., 1992) and replacing it with the coding sequence (CDS) for human heparin-binding epidermal growth factor (HB-EGF), a high-affinity DTR. The HB-EGF/DTR CDS was amplified by high-fidelity PCR from the pRC/CMV/hHB-EGF plasmid (kindly provided by Professor Eisuke Mekada, Osaka University, Japan). The resultant MBP-DTR construct was validated by DNA sequencing to comprise the $1.94 \mathrm{~kb}$ proximal promoter of the mouse myelin basic protein promoter $(M B P$, corresponding to bases -1907 to +36 relative to ATG), the $627 \mathrm{bp}$ human HB-EGF/DTR CDS, and $1.43 \mathrm{~kb} 3^{\prime}$ splicing and the polyadenylation sequence derived from the human $\beta$-globin gene (exon 2, intron 2, exon 3). MBP-DTR plasmid DNA isolated from maxiprep culture (Qiagen) was further purified by CsCl gradient centrifugation. The DNA was digested with ScaI and SphI producing a $1.7 \mathrm{~kb}$ fragment comprising the plasmid backbone and a $4.7 \mathrm{~kb}$ fragment comprising the proximal MBP promoter, human $H B-E G F$ (DTR) CDS, and human $\beta$-globin polyadenylation sequence. The DNA fragments were separated on a $1.5 \%$ agarose (Lonza SeaPlaque; Fisher Scientific)/TBE gel and the $4.7 \mathrm{~kb}$ ScaI/SphI MBP-DTR fragment was extracted from the corresponding gel slice by $\beta$-Agarase I digestion (New England BioLabs). The extracted DNA was dialyzed ("v" series membrane, $0.05 \mu \mathrm{m}$ pore size; Millipore) with microinjection buffer and diluted to $2 \mu \mathrm{g} / \mathrm{ml}$ before microinjecting into the male pronuclei of fertilized C57BL/6 oocytes. Tail biopsies from the offspring of oocyte recipients were assessed for incorporation of the transgene by Southern blot and PCR analysis. For Southern blot analysis, genomic DNA was digested with BstXI followed by agarose gel electrophoresis. Gel blots prepared using nitrocellulose membranes (Hybond $\mathrm{N}+$; GE Healthcare) were hybridized with a ${ }^{32} \mathrm{P}$-labeled DNA probe generated by PCR amplification of a 629 bp product from the MBP-DTR construct that encompassed part of the murine MBP promoter and human DTR sequence (sense primer: $5^{\prime}$-CAGGCCCACATT CATATC-3'; antisense primer: 5' -TCTTCCCTAGCCCCTTGCCTTTCT$\left.3^{\prime}\right)$. PCR genotyping of tail biopsy DNA was performed using primers targeting a $512 \mathrm{bp}$ amplicon encompassing part of the vector between the promoter and HB-EGF CDS and part of the human HB-EGF CDS with $80 \%$ homology to murine HB-EGF (sense primer: $5^{\prime}$-GCTCGAATAATTCTAG GGTC-3'; antisense primer: 5'-GGTCATAGGTATATAAGCGA-3'). Of the 18 founders that exhibited germline transmission of the MBP-DTR transgene, we describe the characterization of one line denoted MBP-DTR Line $100 \mathrm{~A}$ which is hereafter referred to as MBP-DTR mice.

Animal cohorts. All experimental mice were maintained on a pure C57BL/6 background on a $12 \mathrm{~h}$ light-dark cycle and provided with rodent chow (Barastoc Products) and water ad libitum. Experimental cohorts were generated by crossing $M B P-D T R$ heterozygotes with wild-type C57BL/6 to produce heterozygous MBP-DTR and wild-type littermates or, alternatively, by crossing $M B P-D T R$ heterozygotes with Pdgfra-CreER ${ }^{T 2+/+}: R 26 R-Y F P^{+/+}$mice to generate Pdgfra-CreER ${ }^{T 2+/-}$ :
R26R-YFP ${ }^{+/-}: M B P-D T R^{+/-}$and Pdgfra-CreER ${ }^{T 2+/-}:$ R26R-YFP ${ }^{+/-}$: wild-type littermates. Pdgfra-CreER ${ }^{T 2}$ mice (Rivers et al., 2008) and R26RYFP mice (Srinivas et al., 2001) were kindly provided by Professor William D. Richardson (Wolfson Institute for Biomedical Research, University College of London) and Professor Frank Costantini (Columbia University Medical Center, New York), respectively. Experimental procedures were conducted in accordance with National Health and Medical Research Council guidelines and were approved by the animal ethics committees of the Florey Neuroscience Institutes and University of Queensland.

Intraperitoneal injection and gavaging. For the initial experimental cohorts, mice aged $8-10$ weeks were given a single intraperitoneal injection of $200 \mathrm{ng}$ DT $(2 \mu \mathrm{g} / \mathrm{ml}$ in saline; Sigma) or an equal volume $(100 \mu \mathrm{l})$ of saline as control. For subsequent cohorts, DT was administered as a single intraperitoneal injection at a fixed dose of $10 \mu \mathrm{g} / \mathrm{kg}$ (prepared at 1 $\mu \mathrm{g} / \mathrm{ml}$ in saline); vehicle controls received saline at a dose of $10 \mu \mathrm{l} / \mathrm{g}$. Three experimental groups were typically assessed: $M B P-D T R$ mice administered DT, MBP-DTR mice administered saline, and wild-type littermates administered DT. To induce recombination in $P d g f r a-C r e E R^{T 2}$ : R26R-YFP:MBP-DTR and Pdgfra-CreER ${ }^{T 2}: R 26 R-Y F P$ :wild-type mice, tamoxifen (prepared at $40 \mathrm{mg} / \mathrm{ml}$ in $50^{\circ} \mathrm{C}$ corn oil; Sigma) was administered by oral gavage $(300 \mathrm{mg} / \mathrm{kg} / \mathrm{d})$ for 4 consecutive days, commencing $7 \mathrm{~d}$ before DT challenge. Following DT or saline challenge, mice were weighed and monitored daily for signs of neurological impairment. The clinical course was scored as follows: $0=$ healthy, $1=$ pelvis lowered, $2=$ pelvis on ground, $3=$ hindpaw slippage, $4=$ kyphosis, $5=$ tail spasticity, $6=$ upper torso lowered, and $7=$ labored breathing.

Immunohistochemistry. Mice were deeply anesthetized with $100 \mathrm{mg} / \mathrm{kg}$ sodium pentobarbitone then transcardially perfused with PBS followed by $4 \%$ paraformaldehyde (PFA)/PBS. Extracted brains, spinal cords, and sciatic nerves were postfixed in $4 \%$ PFA/PBS for $2 \mathrm{~h}$ on ice, rinsed briefly in PBS, and then equilibrated in 20\% sucrose/PBS for $24 \mathrm{~h}$ before embedding in Tissue-Tek O.C.T. compound (Sakura FineTek) and freezing on an isopentane bath over dry ice. Tissue was stored at $-80^{\circ} \mathrm{C}$ until sectioned. Ten-micron-thick cryosections of the brain (coronal), spinal cord (coronal and longitudinal), and sciatic nerve (longitudinal) were collected onto Superfrost Plus slides (Menzel Gläser) and air-dried for $1 \mathrm{~h}$ before storing at $-80^{\circ} \mathrm{C}$ until stained.

Cryosections were blocked for $1 \mathrm{~h}$ with PBS containing 0.3\% Triton X-100 and 10\% normal serum (goat or donkey depending upon host of secondary antibodies). In some instances serum was replaced with $1 \%$ BSA and $0.1 \%$ gelatin. Primary antibodies diluted in $10 \%$ normal serum/ PBS were applied for $16 \mathrm{~h}$ at $4^{\circ} \mathrm{C}$ or at room temperature. Slides were rinsed three times in PBS (5 min each) before application of secondary antibodies for $30 \mathrm{~min}$ at room temperature. Slides were rinsed again, counterstained with Hoechst $33342(1 \mu \mathrm{g} / \mathrm{ml}$; Invitrogen), and coverslipped with Mowiol mounting medium. For DTR immunohistochemistry, an Alexa Fluor 488-conjugated goat anti-FITC antibody (1:200; Invitrogen) was used as a tertiary antibody after the secondary antibody rinse to amplify the DTR signal.

The following primary antibodies were used: rabbit anti-panneurofascin (1:2000; a generous gift from Professor Peter Brophy, University of Edinburgh), mouse anti-APC/CC-1 (1:100; Calbiochem), mouse anti-ankyrin G (1:200; Santa Cruz Biotechnology), mouse antioligodendrocytes (1:5000, clone NS-1/RIP; Millipore), rabbit anti- $\beta$ amyloid precursor protein (1:300; Zymed), rat anti-PDGFR $\alpha$ (1:500, clone APA5; BD PharMingen), rabbit anti-NG2 (1:200; Millipore), rabbit anti-cleaved caspase-3 (1:200; Cell Signaling Technology), rabbit anti-CD3 (1:200; Dako), rabbit anti-Krox20 (1:200; Covance), rat antiCD11b (1:200; BD PharMingen), mouse anti-glial fibrillary acidic protein (GFAP) (1:200, clone GA5; Millipore), rabbit anti-GFAP (1:500; Dako), goat anti-HB-EGF (1:200; R\&D Systems), rabbit anti-Ibal (1: 1000; Wako Industries), mouse anti-myelin-associated glycoprotein (MAG) (1:1000; Millipore), rabbit anti-MBP (1:200; Millipore Bioscience Research Reagents), mouse anti-NeuN (1:100; Millipore Bioscience Research Reagents), mouse anti-S100 $\beta$ (1:1000, clone SH-B1; Sigma), rabbit anti-Olig2 (1:200; Millipore), mouse anti-Kv1.2 (1:500; NeuroMab), rabbit anti-Nav1.6 (1:100; Alomone Labs), and mouse antiSMI312 (1:1000; Covance). Secondary antibodies raised in goat or 
donkey and conjugated to FITC, TRITC, or NL557 were purchased from Jackson ImmunoResearch or R\&D Systems and used at 1:200 dilution.

Slides were examined using an AxioPlan2 fluorescent microscope (Zeiss), and images were captured using an Axiocam HRc camera (Zeiss) using Axiovision 7.2 software. For comparative analysis of the intensity of myelin protein labeling, photomicrographs were converted to grayscale and analyzed using ImageJ software (National Institutes of Health; $\mathrm{NIH}$ ). Intensity was defined as the average gray value obtained from all pixels within the region of interest above background. Confocal analysis of nodal protein expression was performed using a FluoView FV1000 confocal microscope (Olympus) under a $40 \times$ oil objective using FluoView confocal software (Olympus).

TUNEL. For combined immunohistochemistry and TUNEL, $10-\mu \mathrm{m}-$ thick coronal cryosections of fresh frozen brain tissue were collected onto Superfrost Plus slides, air-dried for $5 \mathrm{~min}$, postfixed in 4\% PFA/PBS for $20 \mathrm{~min}$ at room temperature, and rinsed three times in PBS. Immunohistochemistry was conducted as previously described using TRITCconjugated secondary antibodies then processed for TUNEL using the Fluorescein In Situ Cell Death Detection Kit (Roche) according to the manufacturer's protocol. Slides were counterstained with Hoechst 33342 and coverslipped as described previously. To quantify the extent of apoptosis, the number of TUNEL ${ }^{+}$Hoechst $^{+}$cells in the cerebral cortex and corpus callosum were counted (bregma level $+0.5 \mathrm{~mm}$ ) in MBP-DTR and wild-type mice ( $n=4$ per genotype) at both 5 and $15 \mathrm{~d}$ following DT challenge and expressed as TUNEL ${ }^{+}$cells per millimeter squared. For statistical analysis, the data were analyzed by one-way ANOVA followed by Bonferroni's post hoc tests.

Electron microscopy. Following perfusion/fixation, the lumbar spinal cord and sciatic nerves were postfixed in Karnovsky's buffer $(2.5 \%$ glutaraldehyde, $4 \%$ PFA, $0.1 \mathrm{~m}$ sodium cacodylate, $\mathrm{pH} 7.3$ ) for $16 \mathrm{~h}$ at $4^{\circ} \mathrm{C}$, rinsed three times in PBS, and stored in $0.1 \mathrm{M}$ sodium cacodylate, $\mathrm{pH} 7.3$, at $4^{\circ} \mathrm{C}$ for $24 \mathrm{~h}$. The tissue was postfixed in a solution of $2 \% \mathrm{w} / \mathrm{v}$ osmium tetraoxide and $1.5 \% \mathrm{w} / \mathrm{v}$ potassium ferricyanide and embedded in Spurr's resin. The dorsal funiculus of the lumbar spinal cord and sciatic nerves were sectioned at $0.5 \mu \mathrm{m}$ thickness on an ultramicrotome (Ultracut S; Reichert) in transverse orientation for analysis of g-ratios (dorsal funiculus, sciatic nerve) or longitudinally (dorsal funiculus) for assessment of nodes of Ranvier.

Semithin $(0.5 \mu \mathrm{m})$ transverse sections were stained with methylene blue and imaged at $10,000 \times$ magnification. Fibers within a $121.5 \mu \mathrm{m}^{2}$ region of interest (ROI) within each dorsal funiculus (two ROI assessed per animal, $n=2$ animals per group) or a $3.07 \mathrm{~mm}^{2}$ ROI within each sciatic nerve (four ROI assessed per animal, $n=2$ animals per group) were assessed using ImageJ software (NIH). For each axon, the axonal area excluding the myelin sheath and the entire fiber area including the myelin sheath were independently outlined and the area calculated using the "measurement" function within ImageJ. In addition, as a measure of myelin integrity, the axonal and fiber diameters were measured and used to calculate g-ratios (ratio of axonal diameter to fiber diameter) (Sherman and Brophy, 2005). Results were analyzed using multivariate ANOVA followed by Tukey's post hoc analysis using SPSS 15.0.

For nodal analysis, the ROI in the dorsal funiculus was confirmed by methylene blue staining before collection of ultrathin $(90 \mathrm{~nm})$ sections. Ultrathin sections were postfixed in lead nitrate and urinyl acetate, mounted on copper grids, and examined in an Elmskop 102 transmission electron microscope (Siemens). Photomicrographs were captured at $25,000 \times$ magnification and negatives were scanned to generate highresolution computer images for analysis. Nodes of Ranvier were examined qualitatively for indications of ultrastructural abnormalities. The percentage of nodes within the dorsal funiculi of DT-challenged MBPDTR $(n=2)$ and wild-type $(n=2)$ mice exhibiting paranodal loop eversion was determined on the basis of assessments by two expert myelin biologists who were blinded to the identity of each group.

Somatosensory-evoked potentials. Six DT-challenged MBP-DTR mice at clinical end point and five matching DT-challenged wild-type littermates were anesthetized with an intraperitoneal injection of ketamine $(0.1 \mathrm{mg} / \mathrm{kg})$ and xylazine $(0.01 \mathrm{mg} / \mathrm{kg})$. Depth of anesthesia was checked by the loss of response to rear paw pinch and additional injection of the anesthetic solution was provided as required. The body temperature of animals was maintained at $36.5^{\circ} \mathrm{C}$ using a controlled heating pad. Animals were mounted in a stereotaxic frame (model 962; Kopf Instruments) and the scalp incised longitudinally and retracted to expose the skull. A hole was drilled above the somatosensory cortex (anteroposterior, -0.5 ; lateral, 2.5 ; and dorsal, $1 \mathrm{~mm}$ ) to allow insertion of a borosilicate glass recording electrode filled with $3 \mathrm{~m}$ saline. A second hole was drilled on the opposite side above the contralateral frontal lobe to allow for the insertion of the reference electrode between the skull and the meninges. Stimulating electrodes made of stainless steel (25 g) were inserted at the sciatic notch and connected to a constant current generator (A385; World Precision Instrument). Local field potentials were recorded at $5 \mathrm{kHz}$ and bandpass filtered $(30 \mathrm{~Hz}-1.8 \mathrm{kHz})$. Each animal received 50 sciatic notch stimulations $(5 \mathrm{~mA} ; 0.2 \mathrm{~ms}$; interspike interval, $15 \mathrm{~s}$ ); the onset of the field potential immediately following the stimulation artifact was used to measure the nerve conduction velocity (values are mean \pm SEM).

Measurement of compound action potentials in isolated sciatic nerve. Eight DT-challenged $M B P$-DTR mice at clinical end point and three matching DT-challenged wild-type littermates were overdosed with sodium pentobarbitone $(100 \mathrm{mg} / \mathrm{kg})$. Sciatic nerves were isolated and placed in saline at room temperature. Nerves were coated in liquid paraffin oil, and the proximal and distal ends were placed onto gold-plated fixed electrodes (proximal end, stimulating electrode; distal end, recording electrode $10 \mathrm{~mm}$ from proximal electrode; medial position, grounding electrode) in a humidified $37^{\circ} \mathrm{C}$ chamber. Square-wave stimulating pulses of $100 \mu$ s duration were delivered at $30 \mathrm{~s}$ intervals using a constant-voltage stimulus isolator (PowerLab 4/20; ADInstruments) at progressive intensities from $0.2 \mathrm{mV}$ up to $10 \mathrm{~V}$. Monophasic compound action potentials (CAPs) generated at suprathreshold stimulus intensity $(6 \mathrm{~V})$ were amplified using a differential bioamplifier (Bio Amp; Maclab) and digitized using Scope software (v4.1; ADInstruments). Post hoc analysis was performed using LabChart Reader for Mac (ADInstruments).

\section{Results \\ $M B P-D T R$ mice express DTR exclusively in oligodendrocytes in the CNS}

To assess the consequences of oligodendrocyte ablation in the adult CNS, we generated transgenic mice that express DTR under the regulatory control of the proximal promoter for the $M B P$ gene (see Materials and Methods; Fig. $1 A$ ). We used a $1.94 \mathrm{~kb}$ $M B P$ promoter that had previously been shown to direct transgene expression specifically to the mature oligodendrocyte lineage (Gow et al., 1992; Wrabetz et al., 1998; Emery et al., 2006). Eighteen germline-transmitting MBP-DTR transgenic founders were identified by Southern blot (Fig. $1 B$ ) and PCR analysis (Fig. $1 C$; see Materials and Methods). We performed a series of immunohistochemical analyses to assess the expression pattern of DTR in the adult CNS of MBP-DTR and wild-type littermates. Immunolabeling was conducted using an antibody that specifically detects human HB-EGF/DTR, but not its murine homolog. Of the $M B P-D T R$ lines identified, five lines expressed DTR in 100\% of the oligodendrocytes. Here we characterize the phenotype of one of these five transgenic lines denoted MBP-DTR Line 100A. Assessment of coronal sections of the adult forebrain in MBP-DTR Line $100 A$ mice, referred to hereafter as $M B P-D T R$, revealed that DTR was highly expressed in white matter tracts (Fig. 1D), whereas only low-level, nonspecific labeling was observed in wild-type littermates (Fig. $1 E$ ). We confirmed that DTR was expressed in mature oligodendrocytes in MBP-DTR mice by multilabel immunohistochemistry. Specifically, all CC- $1^{+}$oligodendrocytes identified in sections from MBP-DTR mice were found to coexpress DTR (Fig. $1 F$ ), whereas no DTR was detected in any CC- $1^{+}$oligodendrocytes in wild-type littermates (Fig. $1 G)$. DTR expression in transgenic mice was found not only within the cell bodies of CC- $1^{+}$oligodendrocytes but also within the processes of oligodendrocytes identified by colocalization of 
A

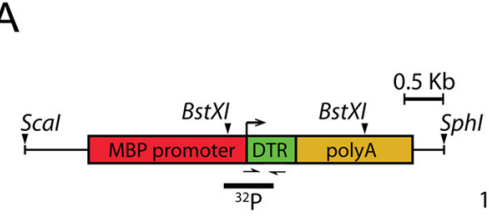

$\mathrm{D}$
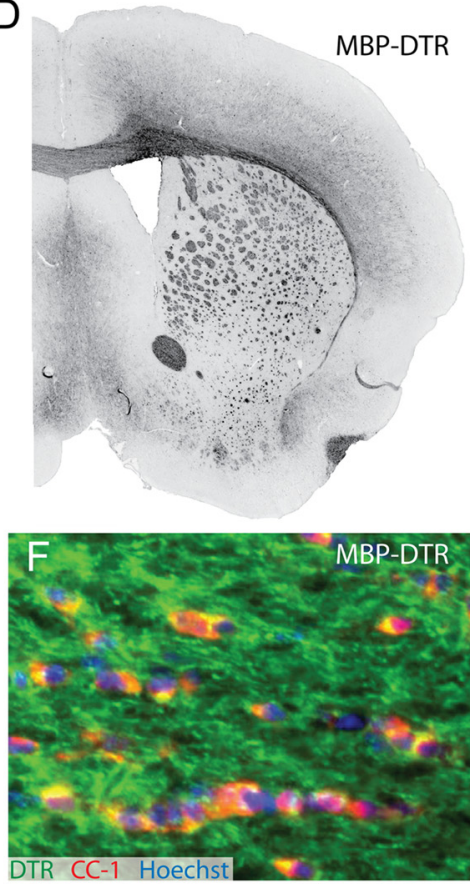

E
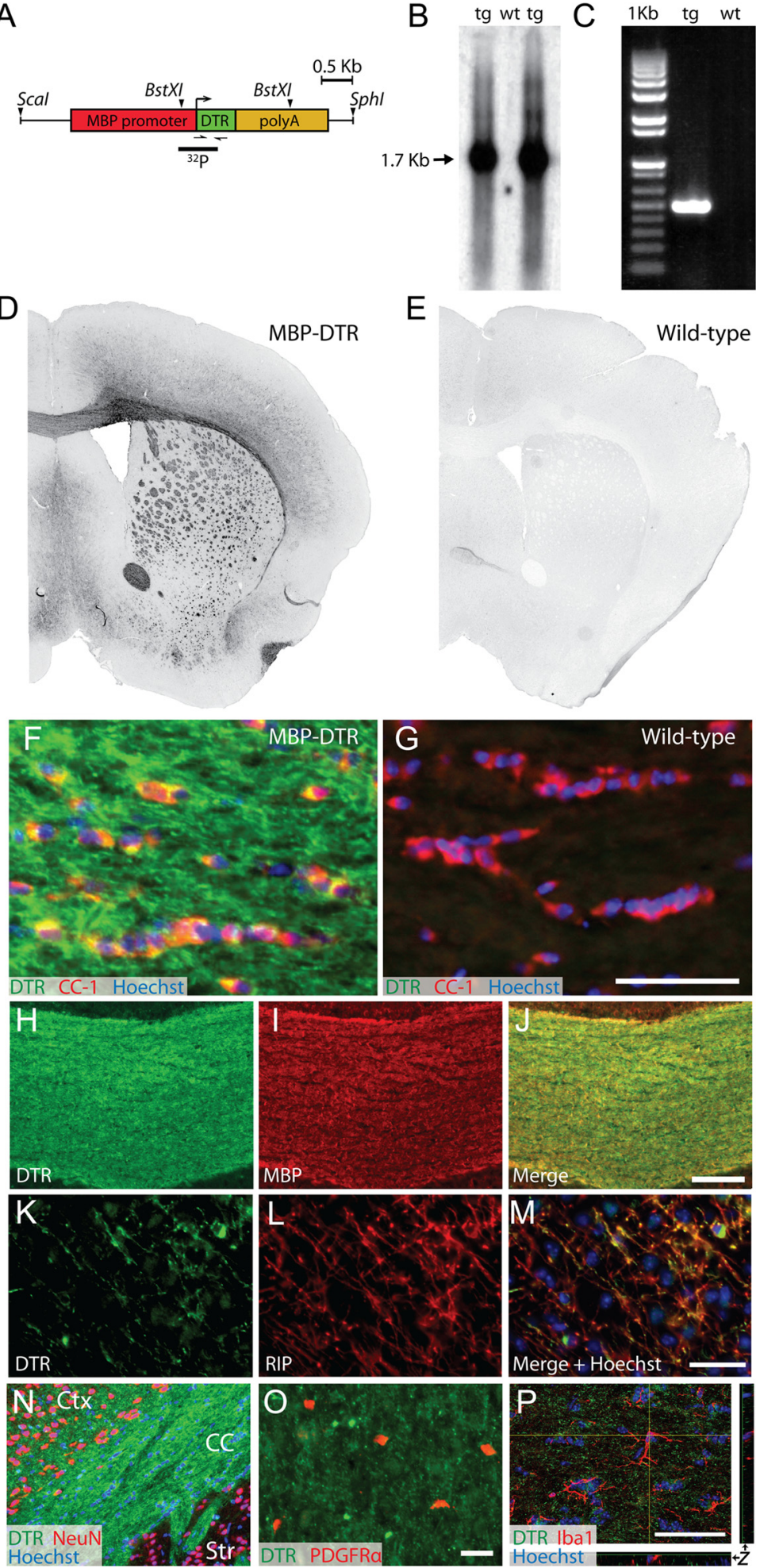

Mesting

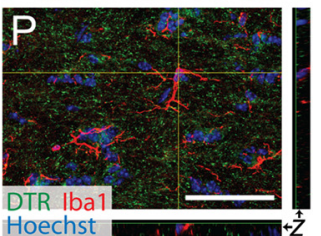

Figure 1. Generation of MBP-DTR transgenic mice expressing DTR within the mature oligodendrocyte population of the adult CNS. A, Schematic representation of the Scal/Sphl-linearized MBP-DTR construct used to generate MBP-DTR founders. The construct comprises the $1.94 \mathrm{~kb}$ proximal promoter of the mouse myelin basic protein promoter, the $627 \mathrm{bp}$ human HB-EGF/DTR CDS, and the $1.43 \mathrm{~kb} 3^{\prime}$ splicing and the polyadenylation sequence derived from the human $\beta$-globin gene. Arrowheads depict the sense and antisense primers used for PCR genotyping. The solid black line indicates the size and position of the ${ }^{32} \mathrm{P}$-labeled probe used for Southern blot analysis of BstXI-digested mouse tail DNA. B, Southern blot analysis of BstXI-digested genomic DNA isolated from mouse tail biopsies used to identify MBP-DTR ( $\mathrm{tg}$ ) founders; note the absence of signal at $1.7 \mathrm{~kb}$ in the wild-type (wt) sample. Hybridization was performed using a 629 bp ${ }^{32}$ P-labeled DNA probe that identified a $1.7 \mathrm{~kb}$ BstXI fragment generated from the MBP-DTR sequence. C, PCR genotyping using primers that amplify a 512 bp product from the MBP-DTR transgene enabled discrimination of MBP-DTR (tg) from wild-type (wt) mice. $\boldsymbol{D}, \boldsymbol{E}$, Immunohistochemical assessment of DTR expression in the forebrain using an anti-human HB-EGF antibody revealed DTR expression within white matter tracts of MBP-DTR (D) but not
DTR and MBP (Fig. $1 H-J$ ) as well as of DTR and RIP (Fig. $1 K-M$ ). In contrast, DTR was not expressed by any other neural cell population examined including $\mathrm{NeuN}^{+}$neurons (Fig. $1 N$ ), PDGFR $\alpha^{+}$oligodendrocyte progenitors cells (Fig. 1O), $\mathrm{Iba}^{+}{ }^{+}$microglia (Fig. $1 P$ ), or $\mathrm{GFAP}^{+}$astrocytes (data not shown). Together, these experiments demonstrate the successful generation of $M B P-D T R$ transgenic mice that express DTR exclusively within the mature oligodendrocyte population of the $\mathrm{CNS}$, in a pattern that closely reflects the expression of myelin proteins.

\section{Severe clinical and motor deficits in MBP-DTR mice following DT challenge} To ablate oligodendrocytes, we administered a single $200 \mathrm{ng}$ dose of DT to MBPDTR transgenic mice $(n=9)$ and wild-type littermates $(n=8)$, or an equivalent volume of saline to MBP-DTR mice $(n=6)$. The two control groups, wild-type mice administered DT and MBP-DTR mice administered saline, did not shown any clinical changes over the subsequent 3 weeks. In contrast, $M B P-D T R$ mice injected with DT exhibited progressive motor deficits that typically began at $9 \mathrm{~d}$ after DT administration and became profound in the next 5-6 d. The maximum survival time following the onset of clinical impairment was $8 \mathrm{~d}$. DT-challenged transgenic animals exhibited hindlimb clasping when held by the tail, an ataxic gait, hindlimb paralysis, tail spasticity, and thoracic kyphosis (Fig. 2A). The phenotype rapidly progressed to include forelimb weakness, respiratory impairment (labored breathing), reduced mobility, lethargy, and weight loss, at which time disabled animals and their matching controls were killed, a time point we have termed the clinical end point. A plot of survival time to clinical end point following DT challenge demonstrates that all MBP-DTR

\section{$\leftarrow$}

wild-type (E) mice. $\boldsymbol{F}, \boldsymbol{G}$, Immunohistochemical assessment of DTR (green) expression among CC-1 (red) immunoreactive oligodendrocytes in the corpus callosum of MBP-DTR $(\boldsymbol{F})$ versus wild-type (G) mice. Nuclei were stained with Hoechst 33258 (blue). $\boldsymbol{H}-\boldsymbol{J}$, DTR expression ( $\boldsymbol{H}$, green) in the corpus callosum colocalized with $M B P(\boldsymbol{I}$, red), as revealed in the merged image (J). $\boldsymbol{K}-\boldsymbol{M}$, DTR expression ( $\boldsymbol{K}$, green) was localized to both the cell body and processes of RIP-labeled oligodendrocytes ( $\boldsymbol{L}$, red), as shown in the merged image $(\boldsymbol{M})$. Nuclei were counterstained with Hoechst. $\boldsymbol{N}-\boldsymbol{P}$, DTR expression (green) was not observed in NeuN-labeled (red) neuronal cell bodies $(\boldsymbol{N})$, in PDGFR $\alpha$-labeled (red) oligodendrocyte progenitor cells $(\boldsymbol{O})$ or in Iba1-labeled (red) microglia ( $\boldsymbol{P}$, confocal stack). CC, corpus callosum, Ctx, neocortex; Str, striatum; $z, z$-axis projection. Scale bars: $\boldsymbol{F}, \mathbf{G}, \boldsymbol{K}-\boldsymbol{P}, 50 \mu \mathrm{m}$; (in J) $\boldsymbol{H}-\boldsymbol{J}, 200 \mu \mathrm{m}$. 
A

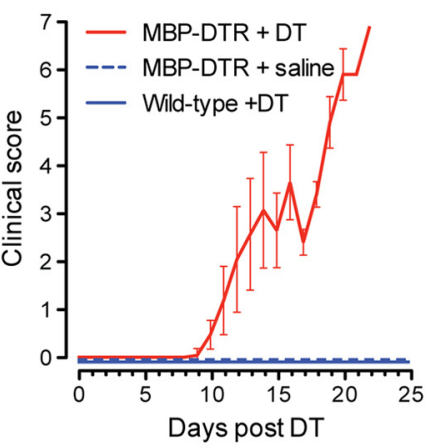

$\mathrm{B}$

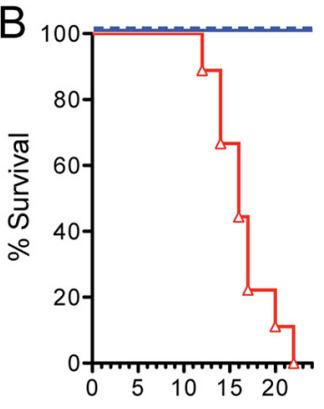

Days post DT
C

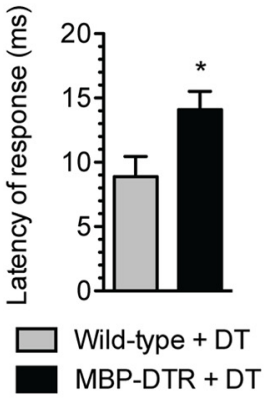

$\mathrm{D}$

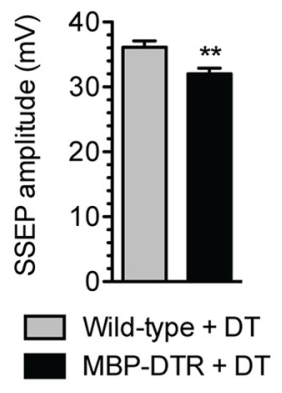

E
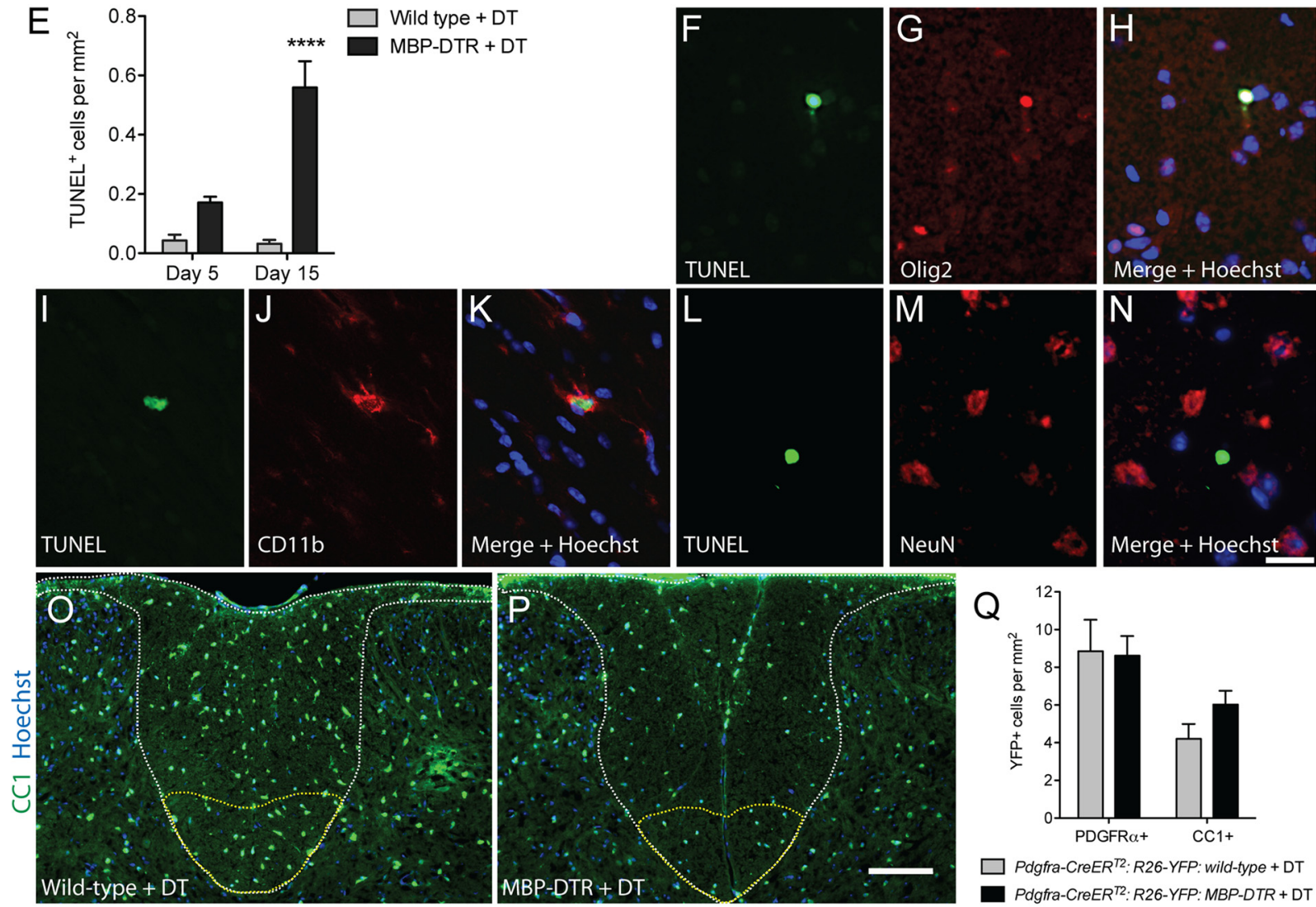

$\square$ Pdgfra-CreERT2: R26-YFP: wild-type + DT

Pdgfra-CreER ${ }^{T 2}:$ R26-YFP:MBP-DTR+DT
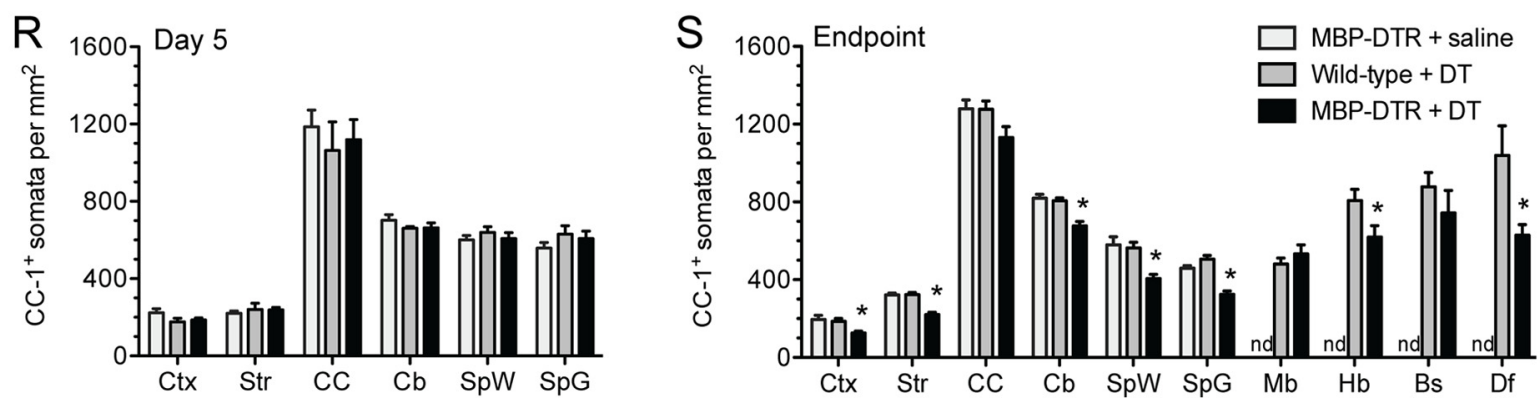

Figure 2. DT-challenged MBP-DTR mice exhibit progressive motor deficits and oligodendrocyte loss. A, MBP-DTR mice challenged with DT developed progressive clinical symptoms from day 9 postchallenge onward (see Materials and Methods for description of clinical scores). Data points represent the average score for all animals $(n=9$ MBP-DTR mice, DT-injected; $n=6$ MBP-DTR mice, saline-injected; $n=8$ WT mice, DT-injected) on each day. The apparent decrease in average disease severity at day 17 reflects the need to kill the most severely affected animals $(n=2)$ on day 16 $(B)$, leaving animals with more moderate disease severity to influence average clinical score. No clinical changes were observed in wild-type controls injected with DT or in MBP-DTR controls injected with saline. $\boldsymbol{B}$, Plot of survival time to clinical end point after DT challenge in MBP-DTR mice. The clinical end point is defined as the time point at which animals were killed due to the severity of the clinical phenotype. C, Latency of sciatic notch-evoked local field potentials measured in the somatosensory cortex was increased by $58.5 \pm 16 \%$ in symptomatic DT-challenged MBP-DTR mice compared with DT-challenged wild-type mice ( $p<0.05$, Student's $t$ test). $\boldsymbol{D}$, Amplitude of sciatic notch-evoked local field potentials measured in the somatosensory cortex was reduced by $11.5 \pm$ $2.39 \%$ in symptomatic DT-challenged MBP-DTR mice compared with DT-challenged wild-type mice ( $p<0.0016$, Student's $t$ test). $E$, Density of TUNEL ${ }^{+}$cells in MBP-DTR versus wild-type controls assessed 5 and $15 \mathrm{~d}$ after DT challenge ( $n=4$ per genotype per time point). Two-way ANOVA revealed an overall effect of genotype $(p<0.0001)$, time $(p=0.005)$, and an interaction between these 2 factors $(p=0.0003)$. Bonferroni's post hoc: MBP-DTR + DT (day 15) versus wild-type + DT (day 15), $p<0.0001$. F-N, Combined labeling for TUNEL and cell type-specific antigens revealed that a subset of TUNEL ${ }^{+}$cells $\left(\boldsymbol{F}, \mathbf{I}, \mathbf{L}\right.$, green) in DT-challenged MBP-DTR mice expressed the oligodendroglial lineage marker Olig2 ${ }^{+}(\mathbf{G}$, red) or the microglial/macrophage (Figure legend continues.) 
mice were killed between 12 and $22 \mathrm{~d}$ after DT injection (Fig. 2 B), with a median survival time of $16 \mathrm{~d}$.

\section{Disabled mice exhibit electrophysiological abnormalities}

To directly assess whether symptomatic MBP-DTR mice challenged with DT exhibited electrophysiological abnormalities, we performed in vivo recordings of somatosensory-evoked potentials (SSEPs) by stimulating at the sciatic notch and recording the response in the somatosensory cortex of both $M B P$ DTR $(n=6)$ and wild-type animals $(n=5)$ challenged with DT. In control animals the onset of the local field potential was detected $8.11 \pm 0.29 \mathrm{~ms}$ after sciatic notch stimulation, whereas in symptomatic MBP-DTR animals SSEPs were recorded $13.42 \pm 0.30 \mathrm{~ms}$ after stimulation, representing a $39.6 \%$ increase in latency (Fig. 2C). Statistical analysis demonstrated that transmission of the SSEP was significantly faster in control versus symptomatic MBP-DTR animals (unpaired Student's $t$ test; $p=0.037$ ). We also observed a statistically significant reduction in the peak amplitude of SSEPs in symptomatic DT-challenged $M B P-D T R$ animals (31.98 $\pm 0.86 \mathrm{mV}$ ) compared with control animals $(36.13 \pm 0.97 \mathrm{mV}, p=0.0016$, unpaired Student's $t$ test) (Fig. 2D).

\section{Induction of oligodendrocyte apoptosis in MBP-DTR mice following DT challenge}

Administration of DT would be expected to result in widespread oligodendrocyte loss throughout the CNS of MBP-DTR transgenic mice. To confirm this, apoptosis within the brains of $M B P$ DTR mice was assessed 5 and $15 \mathrm{~d}$ after administration of DT, corresponding to time points before and during overt clinical disease, respectively ( $n=4$ per time point). Wild-type animals administered DT and assessed 5 and $15 \mathrm{~d}$ postinjection served as controls ( $n=4$ per time point). At day 15, the number of $\mathrm{TUNEL}^{+}$cells in the neocortex of MBP-DTR mice was increased 17.5 relative to wild-type controls (Fig. 2E). Two-way ANOVA of TUNEL $^{+}$cell density identified a significant effect of both genotype $(p<0.0001)$ and time $(p=0.0005)$ and also a significant interaction $(p=0.0003)$ between these two variables. Bonferroni's post hoc analysis confirmed that the increase in apoptotic cell density in MBP-DTR mice compared with wild-type mice at $15 \mathrm{~d}$ post-DT was highly significant $(p<0.0001)$.

We next assessed the phenotype of TUNEL ${ }^{+}$cells and identified two distinct cell types by combined TUNEL and immunohistochemical analysis. Some TUNEL ${ }^{+}$nuclei coexpressed the transcription factor Olig2 (Fig. $2 \mathrm{~F}-\mathrm{H}$ ) suggesting that oligoden-

\section{$\leftarrow$}

(Figure legend continued.) marker $\mathrm{CD} 11 \mathrm{~b}(\boldsymbol{J}$, red) but not the neuronal marker $\operatorname{NeuN}(\boldsymbol{M}$, red) . Merged images with Hoechst 33342 nuclear counterstain (blue) reveal a TUNEL ${ }^{+}$Olig2 ${ }^{+}$cell $(\boldsymbol{H})$ in the cerebral cortex, a TUNEL ${ }^{+} \mathrm{CD}_{11 \mathrm{~b}}{ }^{+}$cell in the corpus callosum $(\boldsymbol{K})$, and a TUNEL ${ }^{+}$ NeuN ${ }^{-}$cell in the cortex ( $\boldsymbol{N}$ ) of an MBP-DTR mouse assessed $15 \mathrm{~d}$ post-DT. $\boldsymbol{O}, \boldsymbol{P}$, Representative photomicrographs of immunolabeling against the oligodendrocyte marker $(C-1$ (green) in the dorsal column (white outline) of wild-type + DT $(\mathbf{O})$ and MBP-DTR + DT (P) mice. The dorsal funiculus is outlined in yellow. Nuclei were counterstained with Hoechst 33342 (blue). Q, Density of YFP ${ }^{+}$cells that are immunoreactive for the oligodendrocyte precursor cell marker (PDGFR $\alpha$ ) or mature oligodendrocyte marker (CC-1) in the white and gray matter of the lumbar spinal cord of DT-challenged Pdgfra-CreER ${ }^{T 2}:$ R26R-YFP:MBP-DTR $(n=4)$ or DT-challenged Pdgfra-CreER $R^{T 2}: R 26 R$-YFP:wild-type $(n=3)$ mice. $R$, S, Density of $C\left(-1^{+}\right.$oligodendrocyte somata at day $5(\boldsymbol{R})$ and at clinical end point (S) in DT-challenged MBP-DTR mice compared with controls. Nd, not determined. Error bars indicate mean $\pm \mathrm{SE}$ of the mean. Cb, cerebellum; $\mathrm{CC}$, corpus callosum; Ctx, cerebral cortex; Str, striatum; SpW, spinal cord white matter; SpG, spinal cord gray matter; $\mathrm{Mb}$, midbrain; $\mathrm{Hb}$, hindbrain; $\mathrm{Bs}$, brainstem; Df, dorsal funiculus of spinal cord white matter. Scale bars: (in $\boldsymbol{N}) \boldsymbol{F}-\boldsymbol{N}, 20 \mu \mathrm{m}$; (in $\boldsymbol{P}) \mathbf{O}, \boldsymbol{P}, 100 \mu \mathrm{m}$. drocytes were undergoing apoptosis following DT challenge. In addition, some TUNEL ${ }^{+}$cells expressed CD11b (Fig. $2 I-K$ ) or Ibal (data not shown), consistent with a microglial/macrophage identity. Notably, we never observed colocalization of TUNEL with $\mathrm{NeuN}^{+}$neuronal nuclei (Fig. $2 L-N$ ), and TUNEL was not identified in $\mathrm{GFAP}^{+}$astrocytes or $\mathrm{NG}^{+}$oligodendrocyte progenitor cells (data not shown). Since DTR expression in MBP-DTR mice is restricted to the mature oligodendrocyte population, the presence of $\mathrm{TUNEL}^{+}$microglia likely reflects an indirect response to the induction of DT-mediated oligodendrocyte apoptosis.

\section{Numbers of CC-1-positive oligodendrocytic cell bodies are reduced in DT-challenged $M B P$-DTR mice}

To assess the extent of oligodendrocyte ablation following DT challenge in MBP-DTR mice, we quantified the density of CC-1 ${ }^{+}$ oligodendrocytes in the forebrain, cerebellum, and spinal cord at $5 \mathrm{~d}$ post-DT injection, and in the forebrain, cerebellum, spinal cord, midbrain, hindbrain, and brainstem at the clinical end point. MBP-DTR mice injected with saline and wild-type mice injected with DT were assessed at the same time points. Representative photomicrographs of CC-1 staining in the dorsal funiculus at the clinical end point are shown (Fig. 2O,P). At the day 5 time point, the number of oligodendrocytes per millimeter squared was not different in any region of the CNS of DTchallenged MBP-DTR mice when compared with controls (Fig. $2 R$; multivariate ANOVA, $p=0.915)$. On the other hand, there was a significant reduction in the total number of CC- $1^{+}$oligodendrocytes in the CNS of the DT-challenged MBP-DTR mice at the clinical end point, with an average reduction in cell density of $26 \pm 1.6 \%$ (multivariate ANOVA, $p=0.030$ ). A region-specific analysis indicated that the numbers of CC-1-positive somata were significantly reduced in the neocortex (Tukey-HSD, $p=$ $0.006)$, striatum $(p<0.001)$, cerebellar white matter $(p=0.001)$, spinal cord white matter $(p=0.001)$, spinal cord gray matter $(p<0.001)$, and hindbrain $(p=0.05)$, but were not significantly reduced in the corpus callosum $(p=0.102)$, midbrain $(p=$ $0.396)$, and brainstem ( $p=0.373$; Fig. $2 S$ ). Interestingly, in the dorsal funiculus of the spinal cord white matter which contains the corticospinal tract there was a $39.5 \pm 5.2 \%$ loss of CC-1positive oligodendrocytes in comparison with DT-challenged wild-type mice ( $p=0.05$; Fig. $2 S$ ).

To assess whether newly differentiated oligodendrocyte precursor cells (OPCs) contribute to the pool of mature oligodendrocytes at clinical end point, we used $P d g f r a-C r e E R^{T 2}: R 26 R-Y F P$ : $M B P-D T R$ transgenic mice and Pdgfra-CreER ${ }^{T 2}: R 26 R-Y F P$ :wildtype littermates to map the fate of OPCs, which all express the PDGFR $\alpha$ receptor, following DT-challenge. Under these conditions we have shown that $52.8 \%$ of all PDGFR $\alpha^{+}$cells express YFP within $7 \mathrm{~d}$ from the first tamoxifen gavage. We found no difference in the density of $\mathrm{YFP}^{+}$cells that were immunoreactive for the oligodendrocyte precursor cell marker, PDGFR $\alpha$ ( $p=$ 0.910; Fig. $2 Q$ ), or mature oligodendrocyte marker, CC-1 ( $p=$ 0.124; Fig. 2Q), in the lumbar spinal cord of $P d g$ fra-CreER ${ }^{T 2}$ : R26R-YFP:MBP-DTR $(n=4)$ or Pdgfra-CreER ${ }^{T 2}: R 26 R-Y F P$ : wild-type $(n=3)$ mice. These findings indicate that OPCs present at the time of oligodendrocyte ablation do not appreciably differentiate into mature oligodendrocytes in compensation for the mature oligodendrocytes that are lost following DT challenge in the MBP-DTR mice. 

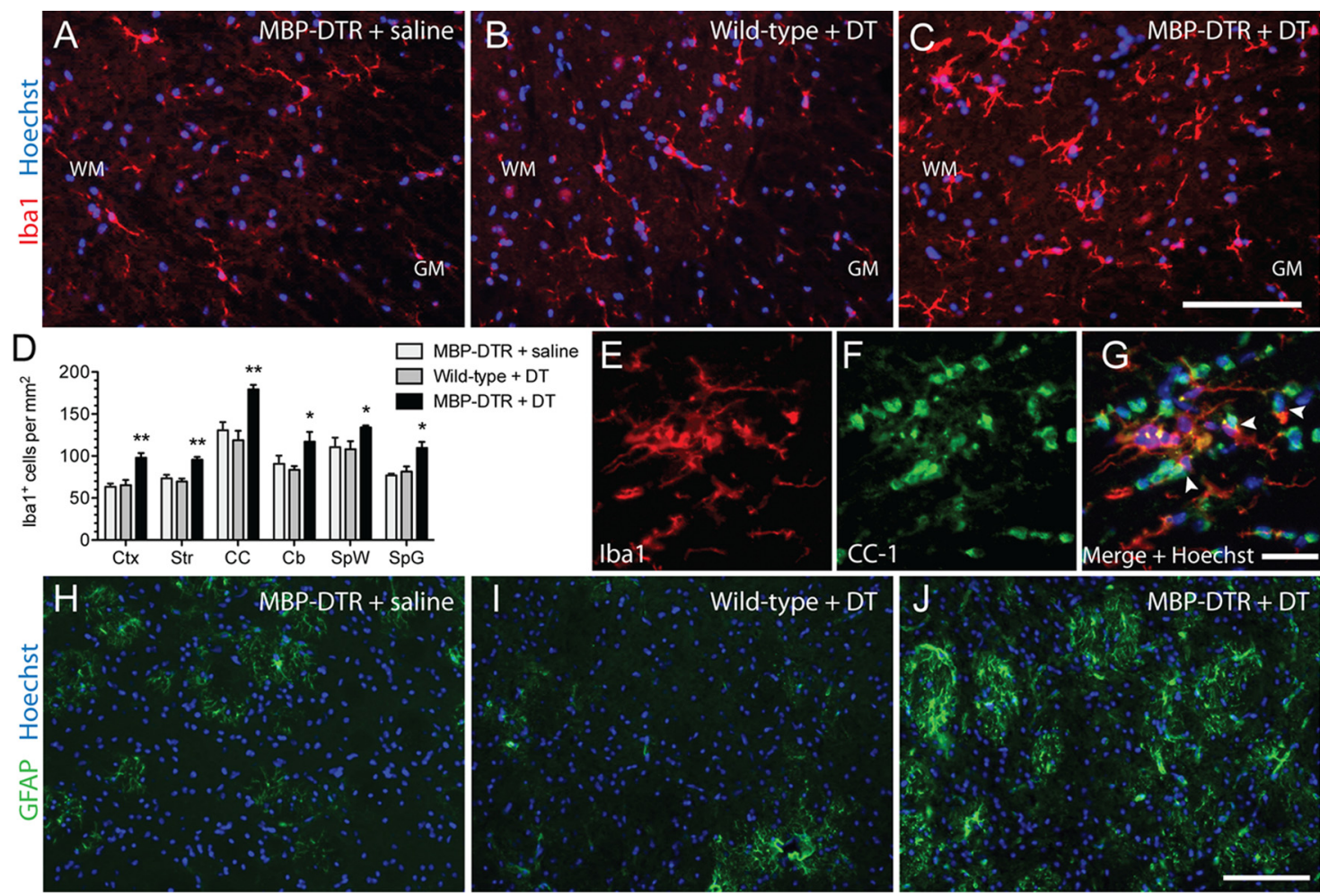

Figure 3. Increased microglial density and GFAP immunoreactivity following oligodendrocyte apoptosis. $A-C$, Immunohistochemistry against the microglial/macrophage marker Iba1 in the ventrolateral aspect of the spinal cord of MBP-DTR + saline $(\boldsymbol{A})$, wild-type + DT $(\boldsymbol{B})$, and MBP-DTR + DT $(\boldsymbol{C})$ mice. MBP-DTR + DT mice were collected at clinical end point and matching controls were examined at the same time point following saline or DT injection. Nuclei were identified with Hoechst (blue). Note the increased intensity of Iba1 staining in the section from a DT-challenged MBP-DTR mouse (C).D, Density of $\mathrm{Iba} 1^{+}$cells in gray and white matter regions of the brain and spinal cord were increased in DT-challenged MBP-DTR mice at clinical end point. E-G, Multicellular aggregates of $\mathrm{Iba} 1^{+}$cells $\left(\boldsymbol{E}\right.$, red) among $\mathrm{CC}-1^{+}$oligodendrocytes $\left(\boldsymbol{F}\right.$, green) assessed 5 dafter DT challenge in MBP-DTR mice. Note the close cell- cell contact between $\mathrm{Iba} 1^{+}$microglia and CC $-1^{+}$ oligodendrocytes (G, arrowheads). $\boldsymbol{H}-\boldsymbol{J}$, Assessment of GFAP immunoreactivity (green) in the ventrolateral aspect of the spinal cord of MBP-DTR + saline $(\boldsymbol{H})$, wild-type + DT $(\boldsymbol{I})$, and MBP-DTR + DT (J) mice reveals evidence of reactive astrogliosis in MBP-DTR + DT mice ( () ) at clinical end point. Scale bars: (in $C, J) A-C, H-J, 100 \mu \mathrm{m}$; (in G) $\boldsymbol{E}-\mathbf{G}, 25 \mu \mathrm{m}$. Cb, cerebellum; CC, corpus callosum; Ctx, cerebral cortex; GM, gray matter; Str, striatum; SpG, spinal cord gray matter; SpW, spinal cord gray matter; WM, white matter.

\section{Reactive changes in microglia/macrophages and astrocytes in disabled MBP-DTR mice}

We next assessed whether oligodendrocyte loss induced an innate immune response by examining Ibal immunoreactivity at the clinical end point in DT-challenged $M B P-D T R$ mice and matched controls (Fig. $3 A-C$ ). Morphological changes in the Iba1-positive microglial cell population were seen in MBP-DTR; DT-challenged mice that were not present in control mice. These changes were characterized by shortened thickened processes and increased Ibal immunoreactivity; however, we did not observe any microglia with the amoeboid morphology typically associated with phagocytic activity (Stence et al., 2001). We also found that the average density of $\mathrm{Iba}^{+}$cells throughout the CNS of DT-challenged MBP-DTR mice relative to DT-challenged wildtype mice was increased by $39 \pm 7 \%$ (Fig. $3 D$; multivariate ANOVA, $p=0.015)$. Examination of specific regions of interest revealed that the density of Iba ${ }^{+}$cells was significantly increased in the neocortex ( $150 \%$ of control; $p=0.001)$, striatum (137\%; $p<0.001$ ), corpus callosum (151\%; $p<0.001)$, cerebellar white matter $(140 \% ; p=0.037)$, and spinal cord white $(124 \%$; $p=$ $0.035)$ and gray matter $(134 \% ; p=0.009)($ Fig. $3 D)$.

We performed similar morphological and quantitative analyses of Iba1 ${ }^{+}$cells at the day 5 time point and observed an overall $35 \pm 10.6 \%$ increase in $\mathrm{Iba}^{+}{ }^{+}$cells specifically in DT-challenged $M B P-D T R$ mice relative to controls; however, this increase was not statistically significant (multivariate ANOVA, $p=0.737$ ). We also assessed the relationship between $\mathrm{Ibal}^{+}$microglia and oli- godendrocytes at this early time point by colabeling for Ibal and CC-1 (Fig. 3E-G). Microglia were frequently in close association with $\mathrm{CC}-1^{+}$somata. Occasional local aggregations of microglia in contact with CC- $1^{+}$oligodendrocyte cell bodies were identified in oligodendrocyte-dense areas such as the corpus callosum and cerebellar white matter (Fig. 3G, arrowheads).

We next assessed for evidence of reactive astrogliosis following oligodendrocyte ablation by qualitative analysis of GFAP immunoreactivity in sections of the brain and spinal cord in DT-challenged $M B P-D T R$ mice at clinical end point and in matched controls. Increased cellular GFAP immunoreactivity was evident in numerous regions, including the neocortex, striatum, and spinal cord (Fig. $3 H-J$ ); although in some regions, most notably the corpus callosum, no such response was identified.

\section{Absence of $\mathrm{CD}^{+}{ }^{+} \mathrm{T}$-cell infiltration into the CNS following oligodendrocyte ablation}

We next sought to assess whether the oligodendrocyte-initiated injury in the MBP-DTR model was associated with the recruitment of peripheral T-cells into the CNS. We examined the brains and spinal cords of DT-challenged MBP-DTR $(n=10)$, salineinjected MBP-DTR $(n=6)$, and DT-challenged wild-type controls $(n=9)$ by immunohistochemical analysis for the T-cell antigen CD3. Animals were assessed both at $5 \mathrm{~d}$ following injection and at the clinical end point. $\mathrm{CD}^{+}$cells were rarely observed in any tissue samples examined. At both time points only one $\mathrm{CD}^{+}{ }^{+} \mathrm{T}$-cell was identified for every two or three tissue 

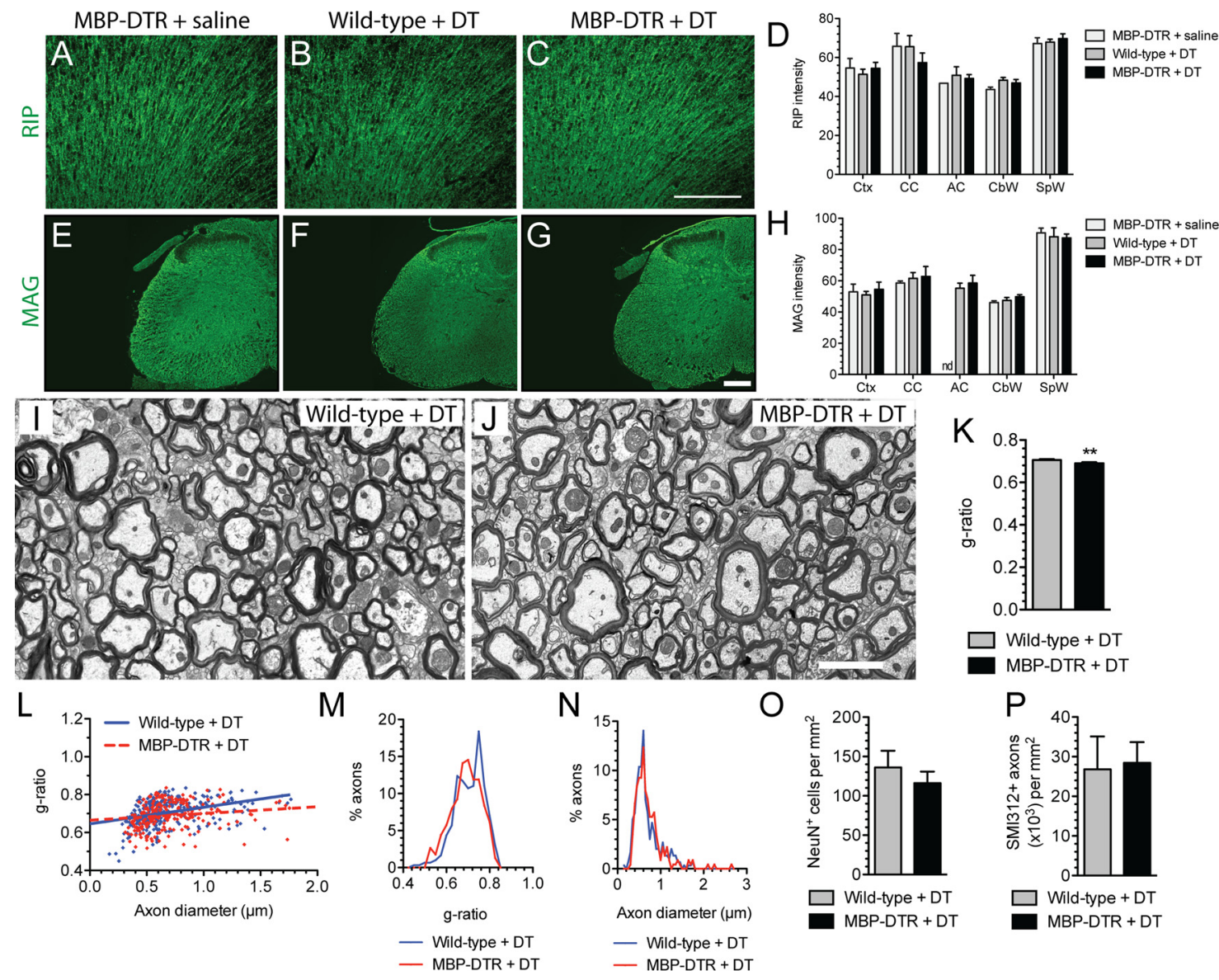

Figure 4. Maintenance of normal levels of CNS myelin and neuronal preservation at clinical end point in symptomatic mice. $\boldsymbol{A}-\boldsymbol{C}$, Immunohistochemistry against the myelin marker RIP (CNPase) on coronal forebrain sections of MBP-DTR + saline $(\boldsymbol{A})$, wild-type + DT $(\boldsymbol{B})$, and MBP-DTR + DT $(\boldsymbol{C})$ mice revealed no distinguishable change in RIP immunoreactivity in the primary motor cortex (M1) among the 3 experimental groups. D, Quantitative analysis of photomicrographs of RIP-labeled CNS tissue revealed no significant change in mean fluorescent pixel density in gray and white matter regions of the brain and spinal cord. $\boldsymbol{E}-\mathbf{G}$, Immunohistochemistry against the myelin marker MAG in spinal cord sections from MBP-DTR + saline $(\boldsymbol{E})$, wild-type + DT $(\boldsymbol{F})$, and $M B P-D T R+$ DT (G) mice revealed no obvious change in immunoreactivity among the 3 experimental groups. $\boldsymbol{H}$, Quantitative analysis of photomicrographs of MAG-labeled CNS tissue revealed no significant change in mean fluorescent pixel density in gray and white matter regions of the brain and spinal cord. $I$, $J$, Electron micrographs of ultrathin transverse sections of the dorsal funiculus of the lumbar spinal cord of wild-type +DT $(\boldsymbol{I})$ and MBP-DTR + DT $(\boldsymbol{J})$ mice at clinical end point revealed normal-appearing myelinated axons. $\boldsymbol{K}$, Comparison of g-ratios for axons in the dorsal funiculus of the spinal cord revealed a $2.1 \%$ reduction in mean g-ratio for axons of DT-challenged MBP-DTR mice versus DT-challenged wild-type controls. $L$, Scatterplot of g-ratio against axonal diameter of neurons in the dorsal funiculus. Linear regression analysis revealed that the slope of the line of best fit was significantly lower for DT-challenged MBP-DTR mice compared with wild-type mice ( $p=0.0057)$. This analysis indicates that the $\mathrm{g}$-ratios of larger caliber axons are reduced in DT-challenged $M B P$-DTR mice relative to controls. $M$, Evidence of a left shift in the frequency distribution of axons with a given g-ratio divided into 0.025 bins suggests moderate swelling of oligodendrocyte membranes at the myelin internode. $N$, Frequency distribution of axon diameters (divided into $0.05 \mu \mathrm{m}$ bins) was similar for DT-challenged MBP-DTR mice and controls indicating no differences in the proportion of axons of a given diameter. $\mathbf{0}$, Immunohistochemical analysis of the spinal cord revealed no difference in the number of neuronal cell bodies $\left(\mathrm{NeuN}^{+}\right.$) per millimeter squared in DT-challenged MBP-DTR versus wild-type mice. Immunohistochemical analysis of the spinal cord revealed no difference in the number of axons (SMI312 ${ }^{+}$) per millimeter squared in DT-challenged MBP-DTR versus wild-type mice. Nd, not determined. Scale bars: (in $\boldsymbol{A}, \boldsymbol{G}$ ) $\boldsymbol{A}-\boldsymbol{C}, \boldsymbol{E}-\boldsymbol{G}, 200 \boldsymbol{\mu m}$; (in J) $\boldsymbol{I}, \boldsymbol{J}, 200$ $\mu \mathrm{m}$. AC, anterior commissure; CbW, cerebellar white matter; CC, corpus callosum; Ctx, cerebral cortex; SpW, spinal cord gray matter.

sections assessed, thus providing no evidence for T-cell recruitment into the CNS in this model.

\section{Maintenance of myelin and neuronal preservation following oligodendrocyte ablation}

Demyelination with neuronal preservation can occur as a result of oligodendrocyte death or dysfunction (Blakemore, 1973; Traka et al., 2010). To investigate demyelination in the MBPDTR model, sections from DT-challenged MBP-DTR mice $(n=$ $7)$ and wild-type counterparts $(n=6)$, as well as saline-injected $\operatorname{MBP}-D T R$ mice $(n=3)$, were analyzed immunohistochemically for expression of the myelin proteins, $2^{\prime}, 3^{\prime}$-cyclic nucleotide $3^{\prime}$ phosphodiesterase (CNPase; Fig. $4 A-C$ ) and MAG (Fig. 4E-G) at clinical end point. CNPase and MAG proteins were chosen for analysis as they are both lost early during experimental demyelination (Felts et al., 2005) and in pattern III lesions in MS (Luc- chinetti et al., 2000; Aboul-Enein et al., 2003; Marik et al., 2007), and are therefore considered robust immunohistochemical markers of demyelination. Quantitative analysis of the fluorescence intensity in photomicrographs using ImageJ revealed no changes in RIP (CNPase) or MAG labeling in any of the analyzed regions of the DT-challenged $M B P-D T R$ transgenic mice when compared with controls (Fig. $4 D, H$; two-way ANOVA, $p=0.728$ and $p=0.602$, respectively). Immunohistochemical analysis therefore failed to provide any evidence of overt demyelination in symptomatic mice.

To further investigate the integrity of the myelin sheath, we examined axial sections of the dorsal funiculus of the lumbar spinal cord using electron microscopic analysis. The dorsal funiculus was selected for ultrastructural analysis since it contains axons integrally involved in the control of motor function and CC $-1^{+}$cell counts revealed a $39.5 \pm 5.2 \%$ reduction in oligoden- 

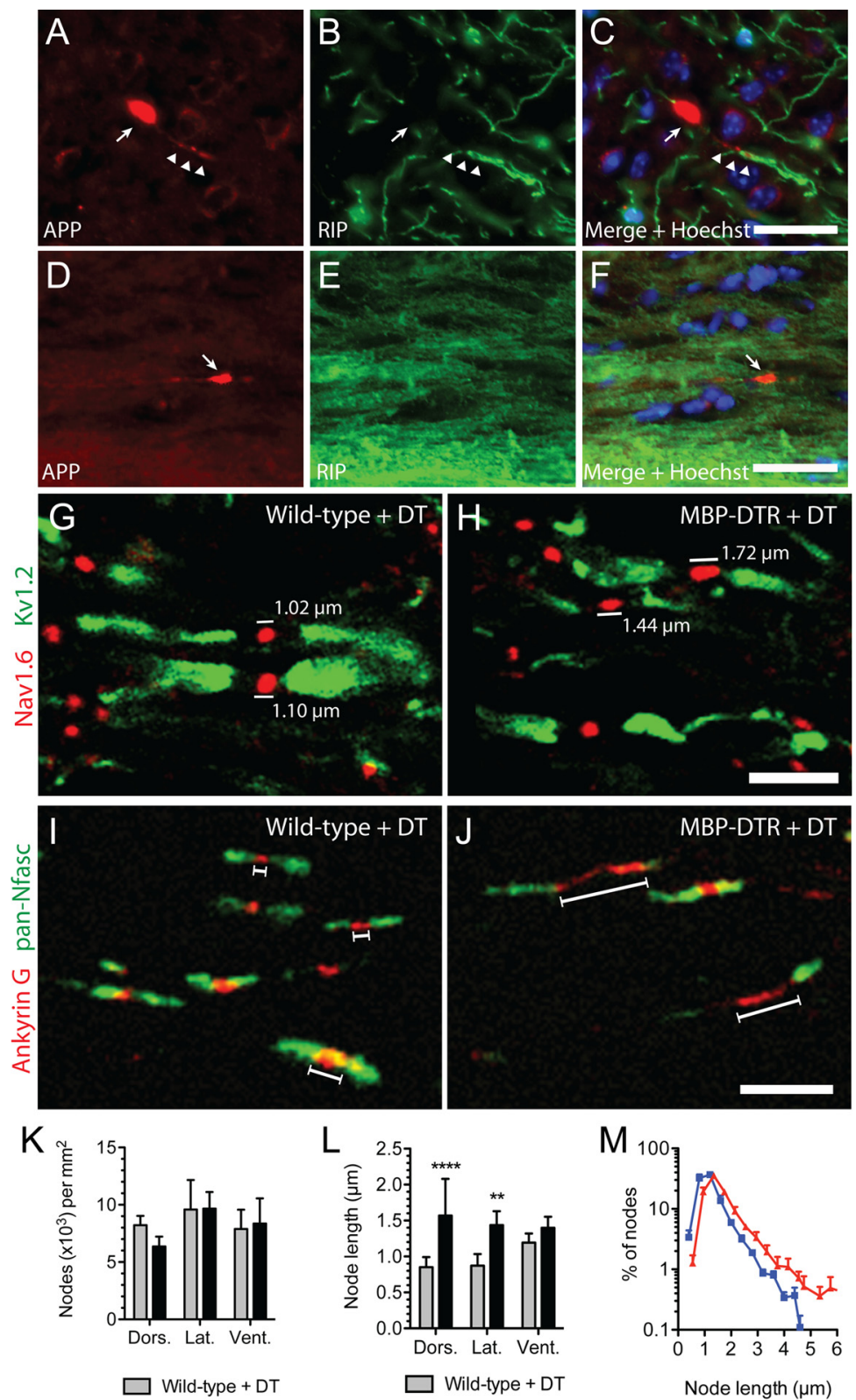

MBP-DTR + DT

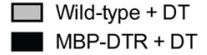

$\mathrm{N}$

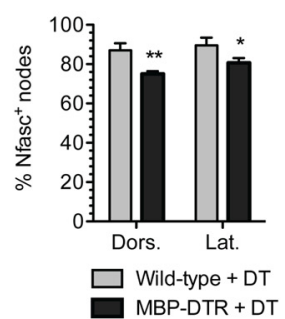

drocyte cell bodies in this region (see above). Sections were prepared from both $\operatorname{MBP}-D T R(n=2)$ and wild-type $(n=2)$ mice challenged with DT and examined at the clinical end point for the MBP-DTR mice (Fig. 4I,J). Measurements of fiber and axon areas were used to calculate g-ratios (Fig. $4 K-M$ ). The mean g-ratio of axons from DT-challenged MBP-DTR mice was $2.1 \%$ less than that of DTchallenged wild-type controls (Fig. $4 \mathrm{~K}$; $p=0.0103$, Mann-Whitney test). Plotting g-ratios against axonal diameters revealed that g-ratios of larger caliber axons were reduced in DT-challenged $M B P$ DTR mice relative to controls (Fig. $4 L$; slope of line of best fit: MBP-DTR + DT, $0.035 \pm 0.014$; wild-type + DT, $0.087 \pm$ $0.013 ; p=0.0057)$. Consistent with these data, frequency distributions of g-ratios revealed a left shift in the population suggestive of moderate swelling of oligodendrocyte membranes at the myelin internode (Fig. 4M). This conclusion was supported by the finding that mean axon diameter was not different in the two groups $(M B P-D T R+D T, 0.72 \pm 0.02$; wild-type $+\mathrm{DT}, 0.68 \pm 0.02 ; p=0.223$, Mann-Whitney test) and the distribution of axonal diameters remained unaltered (Fig. 4N). Collectively, these findings indicate that in regions of oligodendrocyte cell body loss in symptomatic MBP-DTR mice challenged with DT there is no overt demyelination and that compact myelin remains essentially intact (Fig. $4 I, J$ ).

To investigate neuronal loss in the $M B P-D T R$ model, spinal cord sections from DT-challenged $M B P-D T R$ mice $(n=7)$ and wild-type counterparts $(n=$ 6) were analyzed immunohistochemically for expression of neuronal markers (Fig. $4 O, P)$. This revealed no difference in the number of neuronal cell bodies $\left(\mathrm{NeuN}^{+}\right.$, $p=0.4714)$ or the number of axons $\left(\mathrm{SMI} 31^{2+}, p=0.7753\right)$ per millimeter squared in DT-challenged MBP-DTR versus wild-type mice. Similarly, assessment

\section{$\leftarrow$}

$(p<0.0001$, two-way ANOVA) and post hoc analysis confirmed genotype-specific differences in the dorsal funiculus $(p<0.0001)$ and lateral column $(p<0.01$, Bonferroni's post hoc analysis). $\boldsymbol{M}$, Frequency plot of node length demonstrated a right shift in the distribution of node lengths in MBP-DTR + DT mice as opposed to wild-type + DT mice. $\boldsymbol{N}$, Percentage of nodes defined by discrete ankyrin $G$ expression that exhibited bilateral neurofascin (Nfasc) labeling. Two-way ANOVA revealed a significant overall effect of genotype $(p=0.0001)$ and a significant effect of genotype in both dorsal $(p<0.01)$ and lateral $(p<0.05)$ spinal cord (Bonferroni's post hoc analysis). Dors., dorsal; Lat., lateral; Vent., ventral. Data represent mean \pm SEM. Scale bars: (in $\boldsymbol{C}) \boldsymbol{A}-\boldsymbol{E}, 25 \mu \mathrm{m}$; (in $\boldsymbol{J}) \mathbf{G}-\boldsymbol{J}, 5 \mu \mathrm{m}$. 
of axonal density in coronal sections of the hindbrain of DT-challenged MBP-DTR and wild-type mice, a region that exhibited a $23.3 \%$ reduction in $\mathrm{CC}-1^{+}$cell bodies, did not reveal any difference in the density of SMI $312^{+}$axons (data not shown). Collectively these data indicate that functional deficits at the clinical end point are not due to neuronal or axonal loss.

\section{Axonal injury is observed following oligodendrocyte ablation}

The severe clinical phenotype observed in DT-challenged MBP-DTR mice suggests that a significant neuropathology induced by oligodendrocyte cell death could occur even though gross structural integrity and myelination were preserved. To investigate this issue further, we looked for evidence of an underlying axonal pathology in symptomatic DT-challenged $\mathrm{MBP}$ $D T R$ mice. First, we assessed sections for the accumulation of axonal spheroids containing amyloid precursor protein (APP), a robust indicator of acute damage to axonal structure (Trapp et al., 1998). Throughout the CNS of symptomatic DT-challenged transgenic mice, but not controls, $\mathrm{APP}^{+}$axonal spheroids of $\sim 3$ to $5 \mu \mathrm{m}$ in diameter were visible but at very low density (Fig. $5 A, D$; approximately two to three per coronal section). When sections were colabeled with the myelin marker RIP and visualized under high magnification, $\mathrm{APP}^{+}$spheroids were found in regions that remained heavily myelinated (Fig. $5 B, E$ ). Accumulation of APP along the length of myelinated ( $\mathrm{RIP}^{+}$) axons was also observed (Fig. $5 C$ ), indicating that the infrequent observation of axonal pathology was not restricted to rare demyelinated fibers.

\section{Axonal node length is increased and paranodal neurofascin labeling is reduced in the spinal cord of symptomatic MBP- DTR mice}

We next assessed evidence for changes in the expression profile of proteins normally clustered at the node of Ranvier and paranode that are required for fast axonal conduction (Vabnick and Shrager, 1998). Longitudinal sections of the spinal cord of DTchallenged MBP-DTR mice $(n=5)$ and DT-challenged wild-type littermates $(n=4)$ were collected at the clinical end point and immunolabeled for Nav1.6 and Kv1.2 (Fig. 5G,H), proteins normally localized to the node and juxtaparanode, respectively (Poliak and Peles, 2003). In animals treated with DT, we observed an increase in the average length of the Nav1.6 expression domain at the node, suggesting dispersal of sodium channels following DT administration (Fig. 5G, $H$, linear measurements). In contrast, Kv1.2 labeling appeared normal in symptomatic animals.

Given the dispersed pattern of Nav1.6 expression at the node, we next examined the expression of ankyrin G, a structural protein that plays a critical role in clustering voltage-gated sodium channels at the node. To identify nodes, ankyrin G-stained sections were colabeled with a pan-neurofascin (Nfasc) antibody that detects both oligodendrocyte-specific (Nfasc155) and neuron-specific (Nfasc186) isoforms (Tait et al., 2000) (Fig. $5 I, J)$. First, we quantified node density on the basis of Nav1.6 labeling and observed no significant differences between DT- challenged MBP-DTR mice and controls in the dorsal, lateral, or ventral column of the lumbar spinal cord (Fig. $5 \mathrm{~K}$; overall effect of genotype, $p=0.5202$; 2-way ANOVA), consistent with the normal density of SMI $312^{+}$axons previously described. Measurement of the length of the ankyrin G-expressing domain at nodes revealed an increase in node length in symptomatic DTchallenged $M B P-D T R$ animals relative to controls (Fig. $5 \mathrm{~L}$; overall effect of genotype, $p<0.0001$; 2-way ANOVA). Bonferroni's post hoc analysis revealed that relative to controls, node length in DT-challenged MBP-DTR mice was significantly increased in the dorsal funiculus $(p<0.0001)$ and lateral column $(p<0.01)$ but not in the ventral column $(p>0.05)$. To analyze node lengthening in greater detail, the lengths of ankyrin G-labeled nodes in dorsal, lateral, and ventral columns were grouped into $0.4 \mu \mathrm{m}$ bins and plotted to generate a frequency distribution of overall node lengths in the lumbar spinal cord of DT-challenged MBP$D T R$ animals compared with wild-type controls (Fig. 5M). The plot reveals a right-shifted distribution of node lengths for DTchallenged MBP-DTR animals relative to controls. Analysis of the curve of best fit (data not shown) revealed that the two frequency distributions were significantly different (extra sum-of-squares $F$ test, $p<0.0001)$.

We also noted changes in the profile of neurofascin expression in regions outside the ankyrin G-expressing domain, considered to represent the oligodendrocyte-specific Nfasc155 isoform at the paranode. Many nodes in DT-challenged MBP-DTR mice demonstrated almost complete loss of Nfasc155 labeling. This was of particular interest given the integral role of Nfasc155 in establishing axoglial structure at the paranode (Zonta et al., 2008). In contrast, the expression profile of neurofascin within the ankyrin G-expressing domain, likely reflecting the expression of neuronspecific Nfasc186, was preserved. Overall, compared with DTchallenged wild-type controls, symptomatic DT-challenged $M B P-D T R$ mice exhibited a $12 \pm 1.2 \%$ reduction in the percentage of nodes at which Nfasc 155 was present (Fig. $5 N$; overall effect 

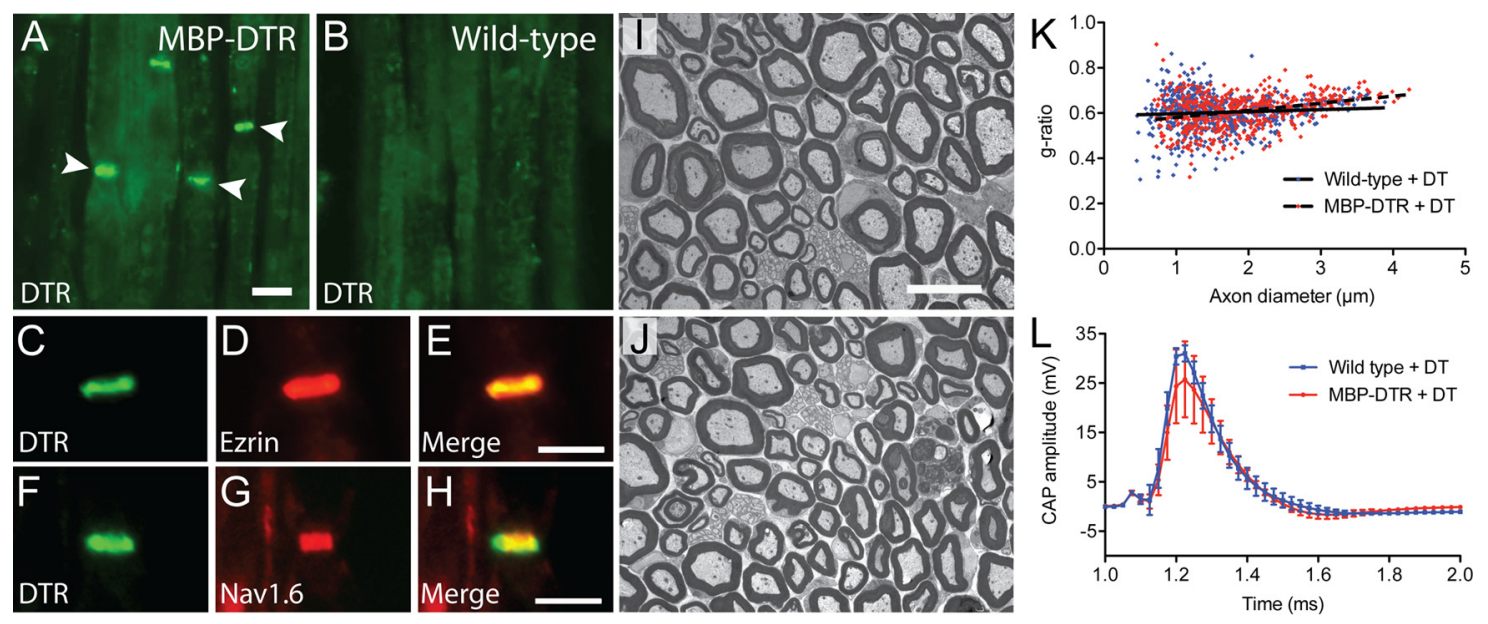

Figure 7. Preservation of peripheral myelination and conduction in the sciatic nerve of MBP-DTR mice. $A, B$, Immunohistochemical analysis of DTR expression in the sciatic nerve of unchallenged $\operatorname{MBP}-\operatorname{DTR}(\boldsymbol{A})$ and wild-type control $(\boldsymbol{B})$ mice revealed restricted foci of DTR protein within the perinodal regions of MBP-DTR nerves ( $\boldsymbol{B}$, arrowheads). $\boldsymbol{C}-\boldsymbol{E}$, Colocalization of perinodal DTR ( $\boldsymbol{C}$, green) with the Schwann cell microvillar marker ezrin $(\boldsymbol{D}$, red). $\boldsymbol{F}-\boldsymbol{H}$, Microvillar DTR expression $(\boldsymbol{F}$, green) in the sciatic nerve surrounds domains of axonal Nav1.6 expression $(\boldsymbol{G}$, red). $\boldsymbol{I}, \boldsymbol{J}$, Electron micrographs of transverse sections of the sciatic nerve of wild-type $+D T(\boldsymbol{I})$ and MBP-DTR $+D T(\boldsymbol{J})$ mice at clinical end point revealed normal-appearing myelinated axons. $K$, $S$ catterplot of $g$-ratio against axon diameter revealed no differences between DT-challenged MBP-DTR mice (red) and DT-challenged wild-type controls (blue). $L$, Electrophysiological assessment of acutely isolated sciatic nerves revealed no significant alteration in peripheral nerve CAPs in DT-challenged MBP-DTR mice versus wild-type mice. Scale bar, (in $A, E, H, I) A-J, 10 \mu \mathrm{m}$.

of genotype: $p=0.0001,2$-way ANOVA; specific effects of genotype: dorsal, $p<0.01$; lateral, $p<0.05$, Bonferroni's post hoc analysis).

\section{Abnormalities of myelination in disabled MBP-DTR mice are restricted to the paranodal region}

To clarify the nature of axonal pathology occurring at the node given the evidence of abnormal protein localization of Nav1.6, ankyrin $\mathrm{G}$, and neurofascin, we assessed nodes of Ranvier at the ultrastructural level. We prepared ultrathin sections from the dorsal spinal cord of two DT-challenged MBP-DTR mice, two DT-challenged wild-type littermates, and one vehicle-treated control. In control DT-challenged wild-type mice and a vehicletreated transgenic mouse, normal nodal structure was maintained (Fig. 6A; wild-type + DT). Paranodal myelin loops were present abutting the node and tightly juxtaposed to the axonal membrane, and the axonal caliber was regular. Some nodes from DT-challenged transgenic mice maintained this regular pattern (Fig. 6B). However, in a significant proportion of nodes examined in DT-challenged MBP-DTR mice, substantial abnormalities were evident with disruption of axonal caliber and accumulation of organelles at both the node and paranode (Fig. 6C,D). There was also evidence that the most proximal paranodal myelin loops (i.e., those closest to the node) were "disconnected" from the axon and everted away from the axon, disrupting the regular structure present at normal nodes (Fig. $6 C, D$, arrowheads). In some cases, paranodes were completely dissociated from the axolemma, reminiscent of the loss of oligodendrocyte-specific neurofascin labeling characterized immunohistochemically. We next extended this assessment to a quantitative analysis of ultrastructural changes in the nodes of Ranvier in longitudinal sections of the dorsal funiculus within the lumbar spinal cord of both vehicle and DT-challenged MBP-DTR mice. We found that $59.4 \%$ of nodes in the DT-challenged MBP-DTR mice exhibited paranodal loop eversion which was never observed in DT-challenged wildtype mice $(n=32 \mathrm{MBP}-\mathrm{DTR}+\mathrm{DT}, n=14$ wild-type $+\mathrm{DT}$ paranodal domains). Collectively, these data indicate that administration of DT leads to significant changes in structure of the nodes and paranodes and the distribution of organizational pro- teins. As a result, there are significant changes in the distribution of ion channels that determine saltatory conduction velocity in these axons.

\section{Peripheral nerve myelination and conduction are unaffected in DT-challenged MBP-DTR mice}

Given that the endogenous MBP gene is expressed not only in oligodendrocytes but also in myelinating Schwann cells (Lemke, 1988) we also assessed the consequences of DT challenge in the peripheral nervous system (PNS) of the MBP-DTR mice. Immunohistochemical staining of the sciatic nerve revealed that there was low-level expression of DTR proximal to the node of Ranvier (Fig. 7A,B). Double labeling confirmed that DTR was localized within perinodal microvilli of Schwann cells defined by expression of ezrin (Fig. $7 C-E$ ), surrounding the domain of axonal Nav1.6 expression (Fig. $7 F-H$ ). To assess whether Schwann cells were ablated following DT challenge, we quantified the density of Krox $20^{+}$cells in the sciatic nerve and found a $33.1 \pm 7.3 \%$ reduction in the number of Krox $20^{+}$Schwann cells at clinical end point $\left(585 \pm 54 / \mathrm{mm}^{2}\right.$ in wild-type + DT mice versus $391 \pm$ $43 / \mathrm{mm}^{2}$ MBP-DTR + DT mice, $n=3$ per group; $p<0.048$, unpaired Student's two-tailed $t$ test). Ultrastructural analysis of the sciatic nerves revealed that myelination is normal in the DTchallenged MBP-DTR mice compared with DT-challenged wildtype (Fig. $7 I, J$ ), consistent with our observations concerning myelination in the CNS. Plotting g-ratio against axon diameter revealed similar distributions for both experimental groups (Fig. $7 K)$ with a mean g-ratio of $0.6085 \pm 0.0036$ in $M B P-D T R+D T$ mice compared with a mean g-ratio of $0.6031 \pm 0.003$ in wildtype + DT mice $(p=0.258$, unpaired Student's two-tailed $t$ test $)$.

To assess peripheral nerve function in these mice we measured compound action potential conduction in isolated sciatic nerves from DT-challenged MBP-DTR and wild-type mice $(n=8$ and three nerves per group, respectively). In contrast to the significant reduction in the velocity and amplitude of central conduction measured by SSEP assessment, no significant alteration in peripheral nerve CAPs was observed in nerves isolated from the DT-challenged MBP-DTR mice (Fig. $7 L$; latency of CAP response: $1.247 \pm 0.009 \mathrm{~ms}$ in MBP-DTR + DT $(n=8)$ compared 
with $1.225 \pm 0.014 \mathrm{~ms}$ in wild-type + DT mice $(n=3) ; p=$ 0.231 , Student's two-tailed $t$ test). Collectively these data suggest that Schwann cells are targeted by DT challenge but that both myelination and peripheral nerve electrophysiological parameters are unaltered. We therefore conclude that Schwann cell targeting in the PNS is likely to be, at most, a minor contributor to the overall phenotype of the DT-challenged mice.

\section{Discussion}

Here we describe the generation and characterization of a novel model of conditional oligodendrocyte ablation using transgenic mice that express DTR in oligodendrocytes throughout the CNS. In response to systemic DT administration, $M B P-D T R$ mice progressively developed profound clinical disability characterized by ataxia, spastic paralysis of the hindlimbs, kyphosis, weight loss, and ultimately respiratory paralysis within $22 \mathrm{~d}$ of DT injection. At clinical end point, mice exhibited the loss of $\sim 26 \%$ of oligodendrocyte cell bodies throughout the CNS but maintained normal g-ratios and normal levels of MAG and CNP proteins indicating no overt demyelination. Schwann cells were also targeted; however, their loss was not associated with abnormalities of peripheral nerve conduction and the animals displayed a spastic rather than a flaccid paralysis, indicative of a central rather than a peripheral clinical deficit. Disabled mice displayed eversion of paranodal loops away from the axolemma and altered distribution of ion channels and structural proteins at both the nodes of Ranvier and paranodes resulting in nodal lengthening. Although axonal density within the spinal cord was unaltered, axonal pathology was evident from APP accumulation within axonal swellings and an increased SSEP latency indicative of reduced conduction velocity. Collectively, these data reveal a critical role for oligodendrocytes in maintaining the structural and functional integrity of axons that is distinct from the provision of myelin.

\section{Comparison with other models of conditional oligodendrocyte ablation}

Previously described binary genetic models of conditional oligodendrocyte ablation were established by crossing two transgenic lines to direct Cre-mediated expression of DT-A or DTR in mature oligodendrocytes (Buch et al., 2005; Traka et al., 2010; Pohl et al., 2011). Despite the different genetic strategies used to induce oligodendrocyte ablation, all transgenic models exhibit a similar clinical phenotype but differ with respect to time of symptom onset and clinical course, with MBP-DTR mice exhibiting the most rapid and severe phenotype. Whereas the former models are associated with overt CNS demyelination and/or myelin vacuolation, DT-challenged MBP-DTR mice exhibited profound clinical disability and axonal pathology before these histopathological features appear. Myelin preservation in symptomatic DTchallenged $M B P-D T R$ mice at clinical end point $(\sim 16 \mathrm{~d}$ post-DT administration) suggests that degenerative mechanisms responsible for degradation of compact myelin following oligodendrocyte apoptosis are not fully induced before the clinical end point. Comparatively, demyelination in MOGi-Cre:iDTR (oDTR) mice (Buch et al., 2005; Locatelli et al., 2012) and PLP/CreER ${ }^{T}$ : ROSA26-eGFP-DTA mice (Traka et al., 2010) was first observed 28 and 35 d postchallenge, respectively, whereas reduced MBP immunoreactivity in PLP-CreER ${ }^{T 2}: R 26-L a c Z / D T-A$ mice was prominent at 39-42 d post tamoxifen (Pohl et al., 2011). The rapid onset of clinical pathology in MBP-DTR mice within 9-12 $\mathrm{d}$ after a single DT injection likely reflects the high-level constitutive expression of DTR by oligodendrocytes that renders the population highly sensitive to DT-mediated cytotoxicity. In contrast, the binary genetic models rely upon repetitive administration of tamoxifen (Traka et al., 2010; Pohl et al., 2011) or DT (Buch et al., 2005; Locatelli et al., 2012) over several days that would be expected to induce an asynchronous course of DTmediated cytotoxicity and cell death rather that the acute toxicity observed in the MBP-DTR model. In addition to differences in kinetics, the percentage depletion of oligodendrocytes across various regions of gray and white matter will reflect the specific Cre drivers and floxed alleles that are used and the precise regimen of tamoxifen or DT that is administered (Saito et al., 2001; Leone et al., 2003).

\section{Conduction impairment is associated with disruption of nodes and paranodes}

The clinical presentation of ataxia and hindlimb paralysis was corroborated by increased latency of SSEP recordings. Structurally, we observed the presence of axonal spheroids at low density throughout the CNS of DT-challenged MBP-DTR mice, a marker of early axonal pathology and transection (Trapp et al., 1998). Ultrastructural analysis revealed disorganization of paranodal structure within spinal cord white matter characterized by eversion of the paranodal loops from the axolemma. Since node and fiber density were unaltered in symptomatic mice, the observation that only a subset of nodes exhibited increased nodal Nav1.6 and ankyrin $\mathrm{G}$ expression suggests that paranodal eversion likely precedes redistribution of Nav1.6 and ankyrin G. Current understanding of paranodal structure and function support this conclusion. Tight axoglial junctions at the paranode form via high molecular weight complexes between the axonal proteins caspr and contactin and oligodendrocyte Nfasc155 (Tait et al., 2000; Bhat et al., 2001). By preventing lateral membrane diffusion, the paranode maintains voltage-gated $\mathrm{Na}^{+}$and $\mathrm{K}^{+}$channels at high concentration at the node and juxtaparanode, respectively, thereby enabling fast saltatory conduction (Sherman and Brophy, 2005). In symptomatic MBP-DTR mice, maintenance of Kv1.2 expression within the juxtaparanode but dispersal of nodal Nav1.6 therefore likely reflects the selective disruption of paranodal loops located closest to the node but preservation of distal loop attachment to the axolemma.

The nodal and paranodal changes observed in MBP-DTR mice bear similarities to defects in a number of myelin and paranodal protein-mutant mice and with changes observed in MS tissue. Notably, mice deficient in Nfasc155 or Caspr do not form normal paranodes and exhibit aberrant clustering of voltagegated ion channels on axons resulting in various motor deficits (Bhat et al., 2001; Rios et al., 2003; Pillai et al., 2009). In MS subjects, Caspr and Nfasc155 are abnormally distributed on myelinated axons that border chronic demyelinated lesions (Wolswijk and Balesar, 2003; Howell et al., 2006) suggesting that changes in paranodal integrity could occur at the leading edge of MS lesion formation before demyelination. Paranodal eversion has also been described in transgenic mice with additional copies of the plp gene (Tanaka et al., 2009) and in CGT mutant mice that have aberrant but viable oligodendrocytes (Dupree et al., 1998). Dispersal of nodal proteins away from the node and paranodal disruption characterized by loss of the axoglial junctions therefore appear to be a common mechanism leading to functional deficits that can occur in isolation or coincident with demyelination. Independent of the initiating event, these pathological changes at the node and paranode are likely to contribute to failure of saltatory conduction and reduced conduction velocity. 


\section{Immune activity and myelination}

The activation, proliferation, recruitment, and conversion of microglia to a phagocytic amoeboid phenotype is an important cellular response that contributes to clearance of myelin debris in various models of CNS demyelination (Merson et al., 2010). In contrast, DT-challenged MBP-DTR mice exhibited only a modest increase in microglial cell density that retained a ramified morphology with evidence of process thickening reminiscent of "prephagocytic" lesions in early MS cases (Barnett and Prineas, 2004), and "predemyelinating" lesions described by Marik et al. (2007). Nevertheless, close apposition between microglia and oligodendrocyte cell bodies $5 \mathrm{~d}$ following DT administration suggests that microglia are responsive to early alterations in oligodendrocyte physiology. Importantly, microglia are capable of phagocytosing damaged cells and membranes without exhibiting an amoeboid morphology (Davalos et al., 2005; Nimmerjahn et al., 2005; Kim and Dustin, 2006) and rapid clearance of apoptotic cell bodies does not stimulate a proinflammatory response in macrophages (Gregory and Devitt, 2004; Town et al., 2005). Thus microglia in MBP-DTR mice could participate in restricted "surgical" phagocytosis of the cell bodies of apoptotic oligodendrocytes without conversion to an amoeboid phenotype required for efficient phagocytosis of compact myelin debris.

It was also notable that there was no adaptive immune response consistent with previous observations in oligodendrocyte ablation models (Buch et al., 2005; Traka et al., 2010; Pohl et al., 2011; Locatelli et al., 2012). The absence of lymphocyte infiltration has also been observed in acute MS lesions (Barnett and Prineas, 2004; Henderson et al., 2009) and therefore does not mitigate against the potential relevance of this model to MS. The essential issue that remains in testing the veracity of Barnett and Prineas' (2004) hypothesis of oligodendrocytopathy as a potential cause of MS is to determine whether there are downstream circumstances in which the adaptive immune system does become activated, as occurs in chronic MS. The recent study by Locatelli et al. (2012), demonstrating that oligodendrocyte cell death failed to potentiate anti-CNS immunity, even in the context of potent induction of T-cell activation and inflammation, suggests that autoimmunity is not readily modulated by oligodendrocyte death. Thus an important issue in addressing Barnett and Prineas' (2004) hypothesis will be to establish how engagement with the immune system differs between models of oligodendrocytopathy that induce T-cell recruitment into the CNS (Ip et al., 2006; Kassmann et al., 2007) and models of oligodendrocyte cell death that do not.

\section{Concluding remarks}

We have demonstrated that oligodendrocyte cell death disrupts the normal structural and molecular integrity of paranodes and nodes of Ranvier, leading to impaired conduction of action potentials and a gross clinical phenotype despite maintenance of normal myelin internodes. The direct expression of DTR in mature oligodendrocytes has many attractive features as a model for oligodendrocyte ablation. Unlike existing binary genetic approaches to ablate oligodendrocytes, the MBP-DTR model provides unlimited freedom to apply Cre/loxP genetics to further explore pathogenic mechanisms. Moreover, this phenotype is elicited with a single dose of DT, enabling clear dissociation of reactive changes that could otherwise occur contemporaneously in the binary genetic models. The MBP-DTR model will facilitate the analysis of the early events involved in the breakdown of the symbiotic relationship between oligodendrocytes and axons. Our results clearly demonstrate that axonal impairment occurs be- fore, or in the absence of, demyelination. This phenotype closely mirrors the early MS lesion, i.e., the prephagocytic lesions described by Barnett and Prineas (2004), as well as the predemyelinating lesions described by Marik et al. (2007) and Pattern III MS lesions (Lucchinetti et al., 2000), providing an opportunity to elucidate the processes that lead to demyelination and axonal degeneration and the relationship between the events. Importantly, the data also serve to illustrate that the genesis of axonal pathology in the absence of demyelination in recent onset disease could be induced by an oligodendrocytopathy.

Oligodendrocytes have been proposed to play an integral role in the maintenance of axonal function by providing trophic support to axons via the provision of energy-rich metabolites to myelinated axons (Nave, 2010). Further evaluation of the MBPDTR model will help clarify the molecular mechanisms that govern oligodendrocyte-axon interactions, and the types of metabolites that might be supplied to axons. Ascertaining the nature of the cytokine profile that predominates in the MBP-DTR model, and contrasting it with that which prevails in demyelinating models could delineate the molecular mechanisms regulating axonal degeneration on the one hand and myelin breakdown and microglial-mediated phagocytosis on the other. Such information could facilitate our understanding of how these various processes are controlled and the circumstances in which it is appropriate to modulate each of them.

\section{References}

Aboul-Enein F, Rauschka H, Kornek B, Stadelmann C, Stefferl A, Brück W, Lucchinetti C, Schmidbauer M, Jellinger K, Lassmann H (2003) Preferential loss of myelin-associated glycoprotein reflects hypoxia-like white matter damage in stroke and inflammatory brain diseases. J Neuropathol Exp Neurol 62:25-33.

Barnett MH, Prineas JW (2004) Relapsing and remitting multiple sclerosis: pathology of the newly forming lesion. Ann Neurol 55:458-468.

Bhat MA, Rios JC, Lu Y, Garcia-Fresco GP, Ching W, St Martin M, Li J, Einheber S, Chesler M, Rosenbluth J, Salzer JL, Bellen HJ (2001) Axonglia interactions and the domain organization of myelinated axons requires neurexin IV/Caspr/Paranodin. Neuron 30:369-383.

Blakemore WF (1973) Demyelination of the superior cerebellar peduncle in the mouse induced by cuprizone. J Neurol Sci 20:63-72.

Buch T, Heppner FL, Tertilt C, Heinen TJAJ, Kremer M, Wunderlich FT, Jung S, Waisman A (2005) A Cre-inducible diphtheria toxin receptor mediates cell lineage ablation after toxin administration. Nat Meth 2:419-426.

Collier RJ (2001) Understanding the mode of action of diphtheria toxin: a perspective on progress during the 20th century. Toxicon 39:1793-1803.

Davalos D, Grutzendler J, Yang G, Kim JV, Zuo Y, Jung S, Littman DR, Dustin ML, Gan WB (2005) ATP mediates rapid microglial response to local brain injury in vivo. Nat Neurosci 8:752-758.

Dowling P, Ming X, Raval S, Husar W, Casaccia-Bonnefil P, Chao M, Cook S, Blumberg B (1999) Up-regulated p75NTR neurotrophin receptor on glial cells in MS plaques. Neurology 53:1676-1682.

Dupree JL, Coetzee T, Blight A, Suzuki K, Popko B (1998) Myelin galactolipids are essential for proper node of Ranvier formation in the CNS. J Neurosci 18:1642-1649.

Emery B, Cate HS, Marriott M, Merson T, Binder MD, Snell C, Soo PY, Murray S, Croker B, Zhang JG, Alexander WS, Cooper H, Butzkueven H, Kilpatrick TJ (2006) Suppressor of cytokine signaling 3 limits protection of leukemia inhibitory factor receptor signaling against central demyelination. Proc Natl Acad Sci U S A 103:7859-7864.

Feigenbaum V, Gélot A, Casanova P, Daumas-Duport C, Aubourg P, DuboisDalcq M (2000) Apoptosis in the central nervous system of cerebral adrenoleukodystrophy patients. Neurobiol Dis 7:600-612.

Felts PA, Woolston A-M, Fernando HB, Asquith S, Gregson NA, Mizzi OJ, Smith KJ (2005) Inflammation and primary demyelination induced by the intraspinal injection of lipopolysaccharide. Brain 128:1649-1666.

Frohman EM, Racke MK, Raine CS (2006) Multiple sclerosis-the plaque and its pathogenesis. N Engl J Med 354:942-955.

Gow A, Friedrich VL Jr, Lazzarini RA (1992) Myelin basic protein gene 
contains separate enhancers for oligodendrocyte and Schwann cell expression. J Cell Biol 119:605-616.

Gregory CD, Devitt A (2004) The macrophage and the apoptotic cell: an innate immune interaction viewed simplistically? Immunology 113:1-14.

Haider L, Fischer MT, Frischer JM, Bauer J, Höftberger R, Botond G, Esterbauer H, Binder CJ, Witztum JL, Lassmann H (2011) Oxidative damage in multiple sclerosis lesions. Brain 134:1914-1924.

Henderson AP, Barnett MH, Parratt JD, Prineas JW (2009) Multiple sclerosis: distribution of inflammatory cells in newly forming lesions. Ann Neurol 66:739-753.

Howell OW, Palser A, Polito A, Melrose S, Zonta B, Scheiermann C, Vora AJ, Brophy PJ, Reynolds R (2006) Disruption of neurofascin localization reveals early changes preceding demyelination and remyelination in multiple sclerosis. Brain 129:3173-3185.

Ip CW, Kroner A, Bendszus M, Leder C, Kobsar I, Fischer S, Wiendl H, Nave KA, Martini R (2006) Immune cells contribute to myelin degeneration and axonopathic changes in mice overexpressing proteolipid protein in oligodendrocytes. J Neurosci 26:8206-8216.

Kassmann CM, Lappe-Siefke C, Baes M, Brügger B, Mildner A, Werner HB, Natt O, Michaelis T, Prinz M, Frahm J, Nave KA (2007) Axonal loss and neuroinflammation caused by peroxisome-deficient oligodendrocytes. Nat Genet 39:969-976.

Kim JV, Dustin ML (2006) Innate response to focal necrotic injury inside the blood-brain barrier. J Immunol 177:5269-5277.

Kuhlmann T, Lingfeld G, Bitsch A, Schuchardt J, Brück W (2002) Acute axonal damage in multiple sclerosis is most extensive in early disease stages and decreases over time. Brain 125:2202-2212.

Lemke G (1988) Unwrapping the genes of myelin. Neuron 1:535-543.

Leone DP, Genoud S, Atanasoski S, Grausenburger R, Berger P, Metzger D, Macklin WB, Chambon P, Suter U (2003) Tamoxifen-inducible gliaspecific Cre mice for somatic mutagenesis in oligodendrocytes and Schwann cells. Mol Cell Neurosci 22:430-440.

Locatelli G, Wörtge S, Buch T, Ingold B, Frommer F, Sobottka B, Krüger M, Karram K, Bühlmann C, Bechmann I, Heppner FL, Waisman A, Becher B (2012) Primary oligodendrocyte death does not elicit anti-CNS immunity. Nat Neurosci. Advance online publication. Retrieved February 26, 2012. doi:10.1038/nn.3062.

Lucchinetti CF, Brück W, Rodriguez M, Lassmann H (1996) Distinct patterns of multiple sclerosis pathology indicates heterogeneity on pathogenesis. Brain Pathol 6:259-274.

Lucchinetti C, Brück W, Parisi J, Scheithauer B, Rodriguez M, Lassmann H (2000) Heterogeneity of multiple sclerosis lesions: implications for the pathogenesis of demyelination. Ann Neurol 47:707-717.

Marik C, Felts PA, Bauer J, Lassmann H, Smith KJ (2007) Lesion genesis in a subset of patients with multiple sclerosis: a role for innate immunity? Brain 130:2800-2815.

Merson TD, Binder MD, Kilpatrick TJ (2010) Role of cytokines as mediators and regulators of microglial activity in inflammatory demyelination of the CNS. Neuromolecular Med 12:99-132.

Nave KA (2010) Myelination and support of axonal integrity by glia. Nature 468:244-252.

Nikić I, Merkler D, Sorbara C, Brinkoetter M, Kreutzfeldt M, Bareyre FM, Brück W, Bishop D, Misgeld T, Kerschensteiner M (2011) A reversible form of axon damage in experimental autoimmune encephalomyelitis and multiple sclerosis. Nat Med 17:495-499.

Nimmerjahn A, Kirchhoff F, Helmchen F (2005) Resting microglial cells are highly dynamic surveillants of brain parenchyma in vivo. Science 308:1314-1318.

Pillai AM, Thaxton C, Pribisko AL, Cheng JG, Dupree JL, Bhat MA (2009) Spatiotemporal ablation of myelinating glia-specific neurofascin (Nfasc NF155) in mice reveals gradual loss of paranodal axoglial junctions and concomitant disorganization of axonal domains. J Neurosci Res 87:1773-1793.

Pohl HB, Porcheri C, Mueggler T, Bachmann LC, Martino G, Riethmacher D, Franklin RJ, Rudin M, Suter U (2011) Genetically induced adult oligo- dendrocyte cell death is associated with poor myelin clearance, reduced remyelination, and axonal damage. J Neurosci 31:1069-1080.

Poliak S, Peles E (2003) The local differentiation of myelinated axons at nodes of Ranvier. Nat Rev Neurosci 4:968-980.

Rios JC, Rubin M, St Martin M, Downey RT, Einheber S, Rosenbluth J, Levinson SR, Bhat M, Salzer JL (2003) Paranodal interactions regulate expression of sodium channel subtypes and provide a diffusion barrier for the node of Ranvier. J Neurosci 23:7001-7011.

Rivers LE, Young KM, Rizzi M, Jamen F, Psachoulia K, Wade A, Kessaris N, Richardson WD (2008) PDGFRA/NG2 glia generate myelinating oligodendrocytes and piriform projection neurons in adult mice. Nat Neurosci 11:1392-1401.

Saito M, Iwawaki T, Taya C, Yonekawa H, Noda M, Inui Y, Mekada E, Kimata Y, Tsuru A, Kohno K (2001) Diphtheria toxin receptor-mediated conditional and targeted cell ablation in transgenic mice. Nat Biotechnol 19:746-750.

Sherman DL, Brophy PJ (2005) Mechanisms of axon ensheathment and myelin growth. Nat Rev Neurosci 6:683-690.

Shults CW, Rockenstein E, Crews L, Adame A, Mante M, Larrea G, Hashimoto M, Song D, Iwatsubo T, Tsuboi K, Masliah E (2005) Neurological and neurodegenerative alterations in a transgenic mouse model expressing human alpha-synuclein under oligodendrocyte promoter: implications for multiple system atrophy. J Neurosci 25:10689-10699.

Srinivas S, Watanabe T, Lin CS, William CM, Tanabe Y, Jessell TM, Costantini F (2001) Cre reporter strains produced by targeted insertion of EYFP and ECFP into the ROSA26 locus. BMC Dev Biol 1:4.

Stence N, Waite M, Dailey ME (2001) Dynamics of microglial activation: a confocal time-lapse analysis in hippocampal slices. Glia 33:256-266.

Tait S, Gunn-Moore F, Collinson JM, Huang J, Lubetzki C, Pedraza L, Sherman DL, Colman DR, Brophy PJ (2000) An oligodendrocyte cell adhesion molecule at the site of assembly of the paranodal axo-glial junction. J Cell Biol 150:657-666.

Tanaka H, Ma J, Tanaka KF, Takao K, Komada M, Tanda K, Suzuki A, Ishibashi T, Baba H, Isa T, Shigemoto R, Ono K, Miyakawa T, Ikenaka K (2009) Mice with altered myelin proteolipid protein gene expression display cognitive deficits accompanied by abnormal neuron-glia interactions and decreased conduction velocities. J Neurosci 29:8363-8371.

Town T, Nikolic V, Tan J (2005) The microglial "activation" continuum: from innate to adaptive responses. J Neuroinflammation 2:24.

Traka M, Arasi K, Avila RL, Podojil JR, Christakos A, Miller SD, Soliven B, Popko B (2010) A genetic mouse model of adult-onset, pervasive central nervous system demyelination with robust remyelination. Brain 133:3017-3029.

Trapp BD, Peterson J, Ransohoff RM, Rudick R, Mörk S, Bö L (1998) Axonal transection in the lesions of multiple sclerosis. N Engl J Med 338:278-285

Trapp BD, Ransohoff R, Rudick R (1999) Axonal pathology in multiple sclerosis: relationship to neurologic disability. Curr Opin Neurol 12:295-302.

Vabnick I, Shrager P (1998) Ion channel redistribution and function during development of the myelinated axon. J Neurobiol 37:80-96.

Weiner HL (2004) Multiple sclerosis is an inflammatory T-cell-mediated autoimmune disease. Arch Neurol 61:1613-1615.

Wolswijk G, Balesar R (2003) Changes in the expression and localization of the paranodal protein Caspr on axons in chronic multiple sclerosis. Brain 126:1638-1649.

Wrabetz L, Taveggia C, Feltri ML, Quattrini A, Awatramani R, Scherer SS, Messing A, Kamholz J (1998) A minimal human MBP promoter-lacZ transgene is appropriately regulated in developing brain and after optic enucleation, but not in shiverer mutant mice. J Neurobiol 34:10-26.

Zonta B, Tait S, Melrose S, Anderson H, Harroch S, Higginson J, Sherman DL, Brophy PJ (2008) Glial and neuronal isoforms of Neurofascin have distinct roles in the assembly of nodes of Ranvier in the central nervous system. J Cell Biol 181:1169-1177. 